\title{
Deformable mapping technique to correlate lesions in digital breast tomosynthesis and automated breast ultrasound images
}

Crystal A. Green a) and Mitchell M. Goodsitt

Department of Nuclear Engineering and Radiological Sciences, University of Michigan, Ann Arbor, MI 48109, USA

Department of Radiology, University of Michigan Health System, Ann Arbor, MI 48109, USA

Kristy K. Brock

Department of Nuclear Engineering and Radiological Sciences, University of Michigan, Ann Arbor, MI 48109, USA

Imaging Physics, University of Texas MD Anderson Cancer Center, Houston, TX 77030, USA

Cynthia L. Davis

GE Global Research, Niskayuna, NY 12309, USA

Eric D. Larson, Jasmine H. Lau, and Paul L. Carson

Department of Radiology, University of Michigan Health System, Ann Arbor, MI 48109, USA

(Received 21 December 2017; revised 22 July 2018; accepted for publication 26 July 2018; published 31 August 2018)

Purpose: To develop a deformable mapping technique to match corresponding lesions between digital breast tomosynthesis (DBT) and automated breast ultrasound (ABUS) images.

Methods: External fiducial markers were attached to the surface of two CIRS multi-modality compressible breast phantoms (A and B) containing multiple simulated lesions. Both phantoms were imaged with DBT (upright positioning with cranial-caudal compression) and ABUS (supine positioning with anterior-to-chest wall compression). The lesions and markers were manually segmented by three different readers. Reader segmentation similarity and reader reproducibility were assessed using Dice similarity coefficients (DSC) and distances between centers of mass $\left(\mathrm{d}_{\mathrm{COM}}\right)$. For deformable mapping between the modalities each reader's segmented dataset was processed with an automated deformable mapping algorithm as follows: First, Morfeus, a finite element (FE) based multiorgan deformable image registration platform, converted segmentations into triangular surface meshes. Second, Altair HyperMesh, a FE pre-processor, created base FE models for the ABUS and DBT data sets. All deformation is performed on the DBT image data; the ABUS image sets remain fixed throughout the process. Deformation was performed on the external skin contour (DBT image set) to match the external skin contour on the ABUS set, and the locations of the external markers were used to morph the skin contours to be within a user-defined distance. Third, the base DBT-FE model was deformed with the FE analysis solver, Optistruct. Deformed DBT lesions were correlated with matching lesions in the base ABUS FE model. Performance (lesion correlation) was assessed with $\mathrm{d}_{\mathrm{COM}}$ for all corresponding lesions and lesion overlap. Analysis was performed to determine the minimum number of external fiducial markers needed to create the desired correlation and the improvement of correlation with the use of external markers.

Results: Average DSC for reader similarity ranged from 0.88 to 0.91 (ABUS) and 0.57 to 0.83 (DBT). Corresponding $\mathrm{d}_{\text {COM }}$ ranged from 0.20 to $0.36 \mathrm{~mm}$ (ABUS) and 0.11 to $1.16 \mathrm{~mm}$ (DBT). Lesion correlation is maximized when all corresponding markers are within a maximum distance of $5 \mathrm{~mm}$. For deformable mapping of phantom A, without the use of external markers, only two of six correlated lesions showed overlap with an average lesion $\mathrm{d}_{\mathrm{COM}}$ of $6.8 \pm 2.8 \mathrm{~mm}$. With use of three external fiducial markers, five of six lesions overlapped and average $\mathrm{d}_{\mathrm{COM}}$ improved to $4.9 \pm 2.4 \mathrm{~mm}$. For deformable mapping of Phantom B without external markers analysis, four lesions were correlated of seven with overlap between only one of seven lesions, and an average lesion $\mathrm{d}_{\mathrm{COM}}$ of $9.7 \pm 3.5 \mathrm{~mm}$. With three external markers, all seven possible lesions were correlated with overlap between four of seven lesions. The average $\mathrm{d}_{\mathrm{COM}}$ was $8.5 \pm 4.0 \mathrm{~mm}$.

Conclusion: This work demonstrates the potential for a deformable mapping technique to relate corresponding lesions in DBT and ABUS images by showing improved lesion correspondence and reduced lesion registration errors with the use of external fiducial markers. The technique should improve radiologists' characterization of breast lesions which can reduce patient callbacks, misdiagnoses and unnecessary biopsies. (C) 2018 American Association of Physicists in Medicine [https:// doi.org/10.1002/mp.13113]

Key words: biomechanical modeling, breast ultrasound, deformable registration, digital breast tomosynthesis 


\section{INTRODUCTION}

Ultrasound imaging used in conjunction with mammography has been shown to improve characterization of breast lesions. ${ }^{1-3}$ Conventional ultrasound imaging is performed freehand in a different geometry (supine) than mammography (upright). Since the acquisition is freehand, the 2D ultrasound images are difficult to reproduce. Automated breast ultrasound (ABUS), visualizes the breast as a 3D image volume and has advantages in terms of reproducibility, acquisition speed and operator independence over conventional breast ultrasound. ${ }^{3-5}$ Likewise, studies have also shown the addition of ABUS imaging used in conjunction with mammography screening for women with dense breasts (ACR3 or ACR4) has significantly improved the breast cancer detection rate with an acceptable recall increase. ${ }^{4-6}$ Digital breast tomosynthesis, digital breast tomosynthesis (DBT), provides better detection and characterization of breast lesions over mammography through the reduction in tissue superposition. However, DBT has not completely eliminated the need for the use of ultrasound. ${ }^{7,8}$ One problem with the use of ultrasound in conjunction with mammography/DBT is that at least $10 \%$ of the time, the lesions found in the ultrasound images do not correspond to those found in mammograms/DBT. ${ }^{9}$

One solution to this problem is to develop a combined $\mathrm{x}$ ray/ultrasound system that images the breast in the same upright geometry using special dual-modality compression paddles. ${ }^{10-18}$ However, the simpler single-sided combined systems are limited in ultrasound depth of penetration, and all studies to date have not completely addressed issues of poor ultrasound transducer coupling at the periphery of the breast in the mammographic geometry. Some improvements such as dual sided ultrasound ${ }^{19}$ for better coverage and resolution have been explored but have not yet been implemented into a single combined system. An alternative to the combined system is to image the breast with the DBT and ABUS modalities in their own separate geometries and then use a deformable mapping technique to relate corresponding lesions. This has the advantage of better acoustic coupling and possibly better coverage of the breast with ultrasound. It utilizes DBT and ABUS systems that are already commercially available and does not require the extra expense of purchasing a special combined system. However, a deformable mapping method has the disadvantage of greater technical difficulty in relating corresponding lesions.

To date, there are no deformable registration techniques for detecting corresponding lesions between ABUS and DBT breast images. The purpose of this study is to investigate the viability of a deformable mapping method to relate corresponding lesions between DBT and ABUS breast images. Such a method should simplify and improve radiologist's characterizations of breast lesions which can reduce patient callbacks, misdiagnoses, and negative biopsies. Using finite element analysis, FEA, a biomechanical algorithm can be used to relate regions of interest between modalities, so a radiologist can directly verify that a lesion seen in a DBT view is solid or cystic as determined from the ABUS image set.
To produce FE based biomechanical models, segmentation of the entire breast volume is required for creation of surface and volumetric meshes. Defining the boundary between breast tissue and the pectoral muscle and distinguishing between glandular, connective, and adipose tissues poses difficulty for segmentation of breast images. Studies have used manual, ${ }^{20,21}$ semi-automated, ${ }^{22,23}$ and automated ${ }^{24-26}$ segmentation in FE-based breast deformation modeling. For large deformations due to breast compressions non-linear models have proven to be more effective than linear elastic models. $^{27}$

Relating breast images across modalities is a challenging task since the breast is a highly deformable entity. Patient positioning in various modalities can change from upright (mammography/DBT), prone (MRI, transmission US, and dedicated breast CT), and supine (ultrasound/ABUS). Immobilization of the breast with compression plates and paddles adds an additional challenge when relating areas of the breast between modalities. A recent study has proposed FE based non-linear biomechanical models for correlating breast structures between two compressed states for cranio-caudal, CC, to medial lateral oblique, MLO, mammography views. ${ }^{28} \mathrm{Sev}-$ eral studies, have found favorable results in using FE modeling between MRI to $\mathrm{x}$ ray registration for CC and/or MLO mammograms. ${ }^{23,24,26,29} \mathrm{~A}$ recent study has found favorable results in relating lesions in supine breast ultrasound to prone MRI. ${ }^{30}$ Another correlation study showed favorable agreement between ultrasound computed tomography and mammography registration. That research group also used ultrasound to determine the distribution in the breast of Young's Modulus from the speed of sound. This distribution was directly used in FE deformation modeling. ${ }^{31}$

Due to high deformation fields of the breast between modalities and even over time in a single modality, ${ }^{32}$ the use of external fiducial markers could provide an added benefit for lesion correlation. Several studies have used external fiducial markers and found improved registration results. In two studies of MRI/PET breast registration, ink was used to mark locations on the breast (up to nine in total). MRI visible markers were placed at those locations prior to MR imaging, and PET-visible markers were placed at the same locations prior to PET imaging. ${ }^{33,34}$ In a study of registration between compressed and uncompressed prone MRI, four external markers were placed around the breast and on the nipple. ${ }^{21}$ Finally, using a volume navigation technique a study of breast MRI and ultrasound registration, three external markers (softgel capsules contain vitamin $\mathrm{E}$ in lipid solution) were used. ${ }^{35}$ In this study, we investigate the use of external fiducial markers to improve the registration of breast lesions in upright DBT to supine ABUS images.

\section{MATERIALS AND METHODS}

\section{A. Phantoms}

Two CIRS Multi-Modality Breast Biopsy and Sonographic phantoms (Computerized Imaging Reference Systems, Inc., 
Norfolk, VA, http://www.cirsinc.com/file/Products/073/073\% 20DS\%20032316.pdf) were used in this study. The first, Phantom A, contains a total of seven lesions: three cystic masses and four dense masses. This phantom also contains three calcifications. The lesions were randomly positioned in a Zerdinebased background gel emulsion and wrapped by a Z-skin membrane material. Z-skin is a proprietary self-healing skinlike membrane that reduces phantom desiccation and simulates the texture and properties of skin during imaging and biopsy. Zerdine is a patented solid elastic water-based polyacrylamide tissue mimicking material that is formulation can be adjusted to correspond to a variety of soft-tissue acoustic properties for ultrasound imaging. ${ }^{36}$ For some time, similar polyacrylamides have been described for ultrasound imaging and therapy phantoms. ${ }^{37,38}$ Since it is a water-based material and the density can be controlled, it can be used for CT and MR imaging as well. $^{36,39}$

The second phantom, Phantom B, contains a total of 12 lesions: six cystic masses and six dense masses. These lesions were randomly positioned in a Zerdine-based background gel, without the Z-skin outer membrane. CT images of both phantoms were acquired with a GE Discovery CT750 CT scanner. These images were used solely to identify lesion type and quantity; the CT scans have no bearing on the deformation algorithm. Material properties for the phantoms were provided by the manufacturer. (See Table I.) HyperMesh, the finite element pre-processor, cannot utilize the Poisson's Ratio of 0.50 for the materials in Table I therefore, a Poisson's ratio of 0.49 was assumed for all materials. For this study, based on visual inspection of the phantom before and after DBT and ABUS imaging there were no external indications that the phantoms did not fully recover to their original states. To better quantitate the differences in phantom deformations, measured load-strain curves are provided in the Data S1. Based on these curves, the elasticities of the phantoms are linear, and phantom $\mathrm{A}$ is a factor of 1.9 times stiffer than phantom B.

\section{B. External fiducial markers}

The present application requires an external marker that does not cause refraction or other distortion below the skin in ultrasound imaging and does not cause artifacts in mammography/DBT imaging. In previous work, Cloutier et al., found glass bead fiducial markers were useful when imaging a multimodality vascular phantom with DSA, CTA, MRA and ultrasound. ${ }^{40}$ We investigated various targets within different background materials as candidates for external fiducial

TABLE I. Phantom material properties.

\begin{tabular}{lcc}
\hline \hline & Young's modulus (E) (kPa) & Poisson's ratio (v) \\
\hline Z-skin membrane & 10 & 0.50 \\
Zerdine background gel & 10 & 0.50 \\
Dense lesions & 60 & 0.50 \\
Cystic lesions & 0 & 0.50 \\
\hline \hline
\end{tabular}

markers. The fiducial marker that we found worked best for both DBT and ABUS was a $1 \mathrm{~mm}$ diameter glass bead target within a clear bubble-free thermoplastic elastomer (TPE) gel. To make this marker, thin samples of TPE were placed on top of the $1 \mathrm{~mm}$ glass beads on a cupcake baking sheet and melted at $130^{\circ} \mathrm{C}$ in a vacuum oven. The vacuum was applied to the TPE as it cooled to eliminate air pockets/bubbles, which can be mistaken for the markers in ultrasound images. The fiducial markers have a thickness of approximately $3 \mathrm{~mm}$.

The markers were placed around the breast, using $\mathrm{Nu}$ Hope Liquid Waterproof Adhesive (Nu-Hope Laboratories, Inc., Pacoima, CA, http://nu-hope.com/products.php). A waterproof adhesive is recommended to ensure maintenance of fiducial marker placement with use of ultrasound coupling gel or lotion. Six external markers were applied to phantom A as show in Fig. 1. Results were obtained using all six markers as well as using five markers (positions F, A, B, C, and $\mathrm{E}$ ), four markers (positions $\mathrm{F}, \mathrm{E}, \mathrm{B}$, and $\mathrm{C}$ ), three markers (position $\mathrm{F}, \mathrm{A}, \mathrm{B}$ ), and two markers (positions $\mathrm{F}$ and $\mathrm{B}$ ). Results for Phantom A, indicated no statistical difference in lesion correlation when using six markers and when using a maximum of three markers at locations F, A, and B. Therefore, for Phantom B, only three markers at those approximate locations were used when imaged.

\section{C. Data collection}

A GE-SenoClaire DBT system (GE Healthcare, Milwaukee, WI) was used to image Phantom A in the CC view with a compressive force of $6 \mathrm{daN}$ and compressed breast thickness of $48 \mathrm{~mm}$. The maximum uncompressed breast phantom thickness measured $98 \mathrm{~mm}$ from reconstructed CT images. Thus, phantom A was compressed 51\% for this study. Six of the seven lesions in this phantom were identified in the reconstructed DBT images. A GE Senographe Pristina DBT system (GE Healthcare, Milwaukee, WI) was used to image Phantom B in the $\mathrm{CC}$ view with a compressive force of $3 \mathrm{daN}$ and compressed breast thickness of $31 \mathrm{~mm}$. For Phantom $\mathrm{B}$, seven of the 12 lesions were identified in the

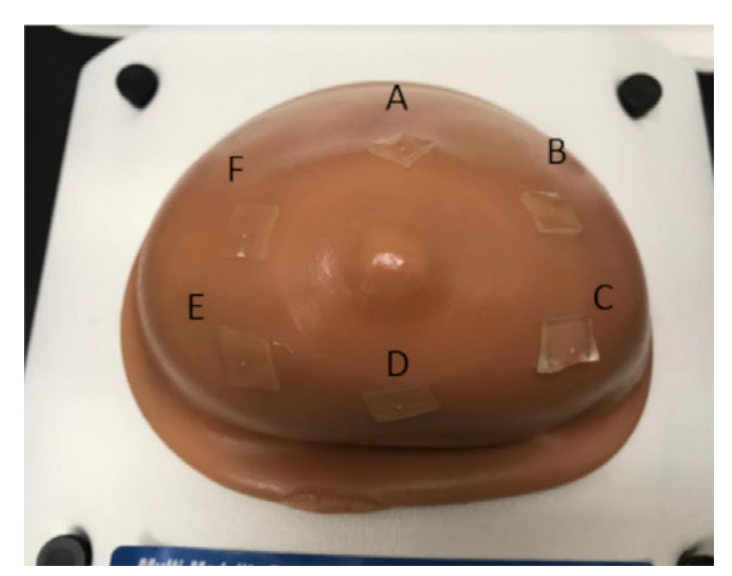

Fig. 1. Phantom A with external fiducial marker locations indicated by A-F. [Color figure can be viewed at wileyonlinelibrary.com] 
reconstructed DBT images. The maximum uncompressed breast phantom thickness measured $97 \mathrm{~mm}$ from reconstructed CT images. Thus, phantom B was compressed $68 \%$ for this study. The reason one lesion in Phantom $A$ and five lesions in Phantom B were not seen in the reconstructed DBT images is that the plastic backing on both phantoms restricted the ability to image close to the "chest wall" where those lesions were located. Figures 2(a) and 2(b), show DBT images of both phantoms including some of the lesions and an external fiducial marker (red arrow).

Both phantoms were imaged with a GE Invenia ABUS system $^{41}$ (GE Healthcare, Milwaukee, WI) immediately after DBT imaging. Light AP compression is applied to the phantoms when they are imaged with the Invenia ABUS system. There are three settings on the system (high, medium, and low) based on imaging depth. The high setting was used for imaging Phantom A and the medium setting was used for imaging Phantom B. The system has a safety stop at $20 \mathrm{lbs}(8 \mathrm{daN})$, which is lower than the compression used in most mammography exams today. The Invenia ABUS reverse curve transducer has a bandwidth of six to $15 \mathrm{MHz}$ and can image up to $50 \mathrm{~mm}$ in depth. The transducer has a width of $153 \mathrm{~mm}$ and automatically travels approximately $170 \mathrm{~mm}$ across a mesh compression paddle. The breast/phantom was positioned supine with anteriorposterior compression applied by the mesh paddle. One ultrasound volume of each phantom was taken in the anterior posterior view. Clinical procedures typically include acquisitions of three separate volumes per breast to ensure full coverage of the breast and axillary region. Raw data from the Invenia ABUS system do not include correction for the curved transducer. Therefore, an algorithm was developed and used to correct for transducer curvature in ABUS images. Figures 2(c) and 2(d), indicate marker positioning under the ABUS imaging for Phantoms $\mathrm{A}$ and $\mathrm{B}$ with the correction for the transducer curvature. In Phantom $\mathrm{A}$, the three calcifications were not seen in the reconstructed ABUS images. A disadvantage of ultrasound imaging is that microcalificatons are often not seen and even larger calcium macrocalcifications deposits can be missed. ${ }^{42}$ All 12 lesions were seen in the ABUS scans of Phantom B.

\section{D. Manual segmentation}

All lesions and external markers from the acquired image sets from both modalities were manually segmented using a free-hand drawing tool within $3 \mathrm{D}$ Slicer ${ }^{43}$ by three readers (CAG, MMG and JHL) using the same window-level settings on the same work station. Readers viewed the images together and agreed upon a window-level setting to be used for each image set to eliminate window-leveling as a variable in interreader concordance of lesion segmentation. Since DBT images have poor axial resolution, the lesions were segmented until the superior and inferior extents of the lesions exhibited significant blur along the edges. These decision points are very subjective so the axial extents of the lesion segmentations in DBT can vary significantly between readers.

Figure 3, shows examples of lesion segmentation for Phantom A and B in both DBT and ABUS image sets. Dice similarity coefficients (DSC) (twice the overlap of corresponding segmented volumes/sum of those volumes) were used to evaluate similarities between lesion and marker contours between readers. ${ }^{44}$ For the DBT and ABUS data sets, one reader manually segmented the outer skin layer from the air and the body of breast phantom $\mathrm{A}$ and segmented the body of breast phantom $\mathrm{B}$ from the air. For the ABUS images, that same reader (a)

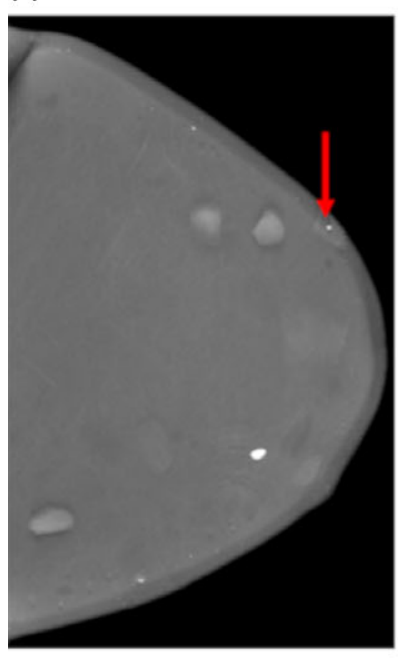

(b)

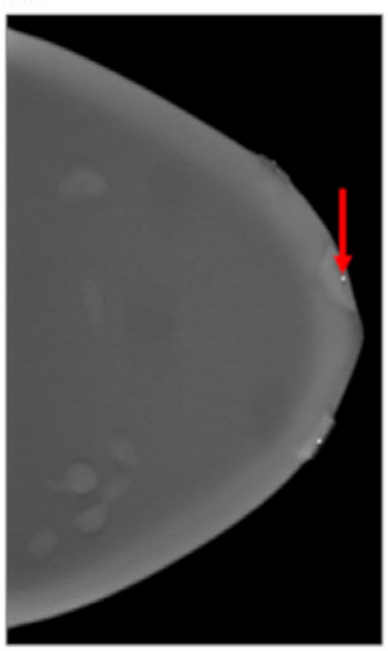

(c)

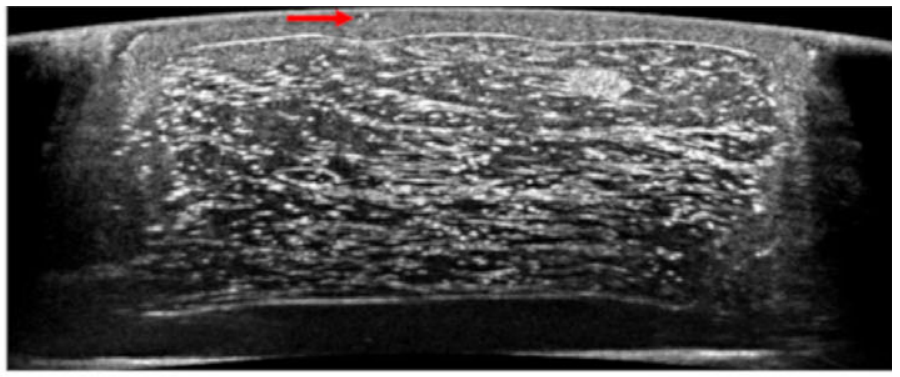

(d)

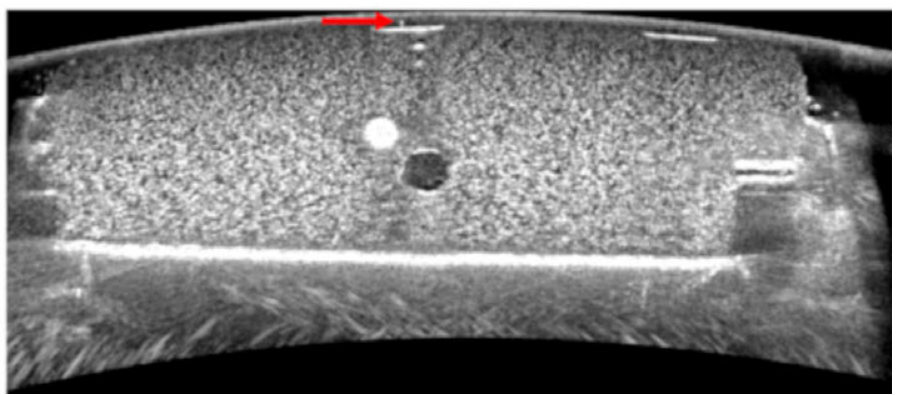

FIG. 2. Digital breast tomosynthesis (DBT) and automated breast ultrasound (ABUS) acquired images. The red arrows indicate the locations of external fiducial

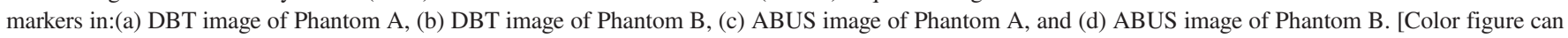
be viewed at wileyonlinelibrary.com] 

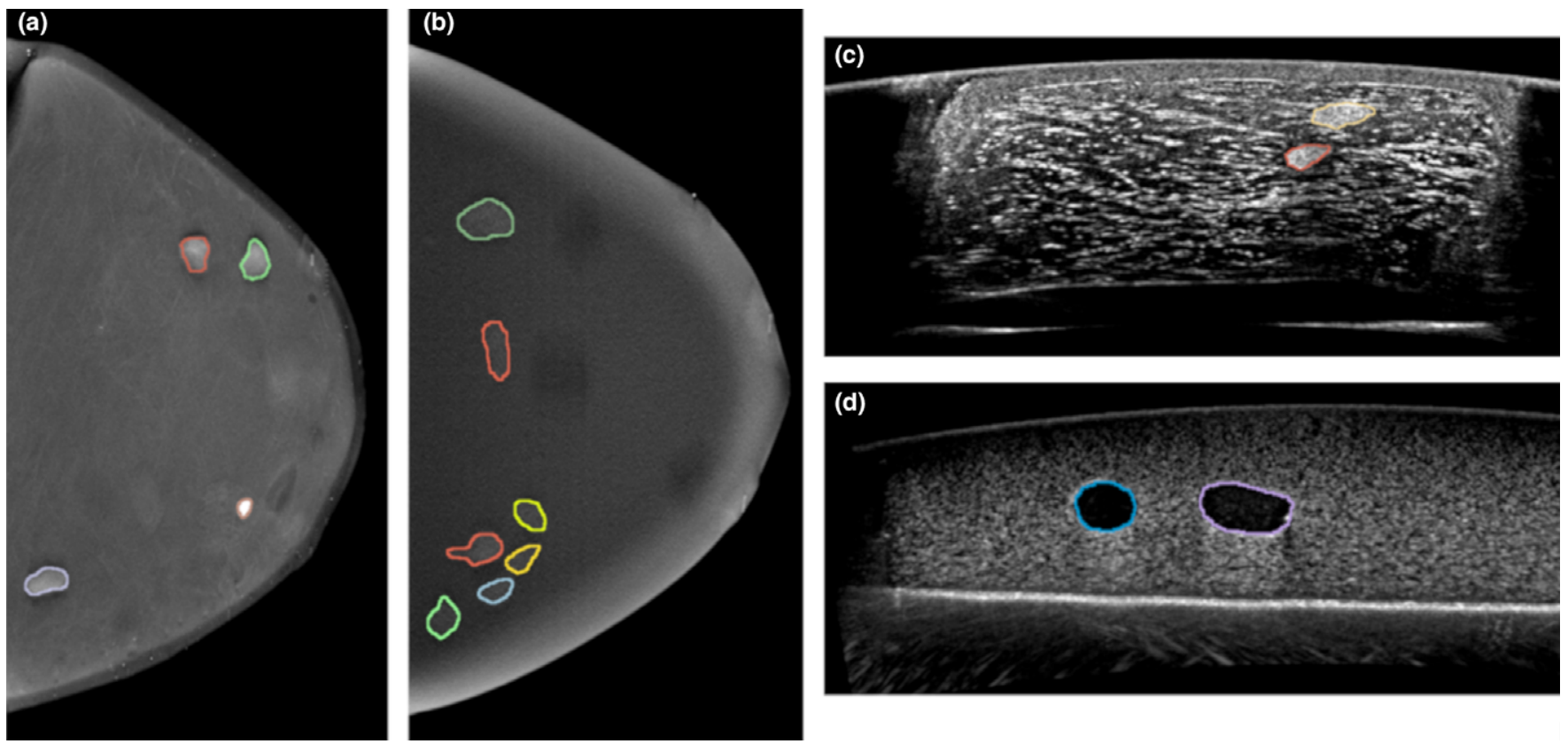

FIG. 3. Reader 1's manual segmentation of a slice in (a) Phantom A — digital breast tomosynthesis (DBT), (b) Phantom B — DBT, (c) Phantom A — automated breast ultrasound (ABUS) (d) Phantom B - ABUS. [Color figure can be viewed at wileyonlinelibrary.com]

performed these same segmentations manually. There was no correction in the segmentations for DBT reconstruction artifacts. After segmentations were completed, resampling was used to decrease runtime when converting segmentations into triangular surface meshes. The DBT images were resampled from a native voxel size of $0.1 \mathrm{~mm}$ width, $0.1 \mathrm{~mm}$ length, and $1.0 \mathrm{~mm}$ depth to $0.2 \mathrm{~mm}$ width, $0.2 \mathrm{~mm}$ length, and $1.0 \mathrm{~mm}$ depth. The ABUS images were resampled from a native voxel size of $0.082 \mathrm{~mm}$ width, $0.2 \mathrm{~mm}$ depth, and $0.506 \mathrm{~mm}$ length (distance between adjacent slices), to $0.2 \mathrm{~mm}$ width, $0.2 \mathrm{~mm}$ depth, and $0.506 \mathrm{~mm}$ length.

\section{E. Deformable mapping algorithm}

The deformable mapping algorithm is an automated process that integrates the use of Morfeus, a commercial FE pre-processor (HyperMesh 2017, Altair Engineering, Troy, MI) and a finite element analysis (FEA) solver processor (Optistruct 2017, Altair Engineering, Troy, MI). An overview of the process is shown in Fig. 4. The entire deformable mapping algorithm takes up to approximately $40 \mathrm{~min}$ to complete from start to finish on a Windows 7 Intel $^{\circledR}$ Core $^{\mathrm{TM}}$ i7 CPU with a speed of $2500 \mathrm{MHz}$ and $4 \mathrm{~GB}$ RAM. In ABUS imaging, poor acoustic contact with the transducer often occurs around the periphery of the breast causing artifacts as shown on the right and left sides of Figs. 2(c) and Fig. 2(d). These artifacts cause the actual breast size and shape in ABUS imaging to be not as well defined in comparison to DBT. An example of the segmentation of the skin layer for both phantoms is shown in the Data S1. Reader bias can affect the segmentation in the periphery boundary. The use of external markers for registration should help to reduce and correct for these differences in lesion registration. On the other hand, the breast shape and size are better defined in the DBT images as shown in Figs. 2(a) and 2(b). Therefore, we chose to have the deformable mapping algorithm perform deformation only to the DBT-FE model and register the resulting deformation to the ABUS image set. The external fiducial markers allow for adjustments to be made since all markers observed in the ABUS images had potentially corresponding points in the DBT images. More sophisticated registration rules are required if that is not the case.

\section{E.1. Conversion of DICOM segmented images to triangular surface mesh}

Morfeus, a FE based multi-organ deformable image registration platform, converts all DICOM segmented contours into individual triangular surface mesh for use in the FE model pre-processor. ${ }^{45}$ Morfeus converts the DICOM image data into mask files for image analysis using Interactive Data Language, Research Systems Inc. (Boulder, CO, USA). Each mask file is then converted into a triangular surface mesh file. Within HyperMesh, the shrinkwrap function is used on the Morfeus generated triangular surface element mesh to create a trias surface mesh for further processing and FEA. Prior to FEA, an element quality check is performed to ensure all elements in the model are within various specifications (e.g., aspect ratio, Jacobian, warpage etc.) in order for the analysis to commence. Therefore, Morfeus uses Laplacian smoothing and a decimate function to better ensure the triangular mesh is within element quality specifications by smoothing rough or sharp elements to ensure viable mesh integrity while maintain a reasonable mesh size to maintain structural features. 


\section{Surface Mesh Creation}
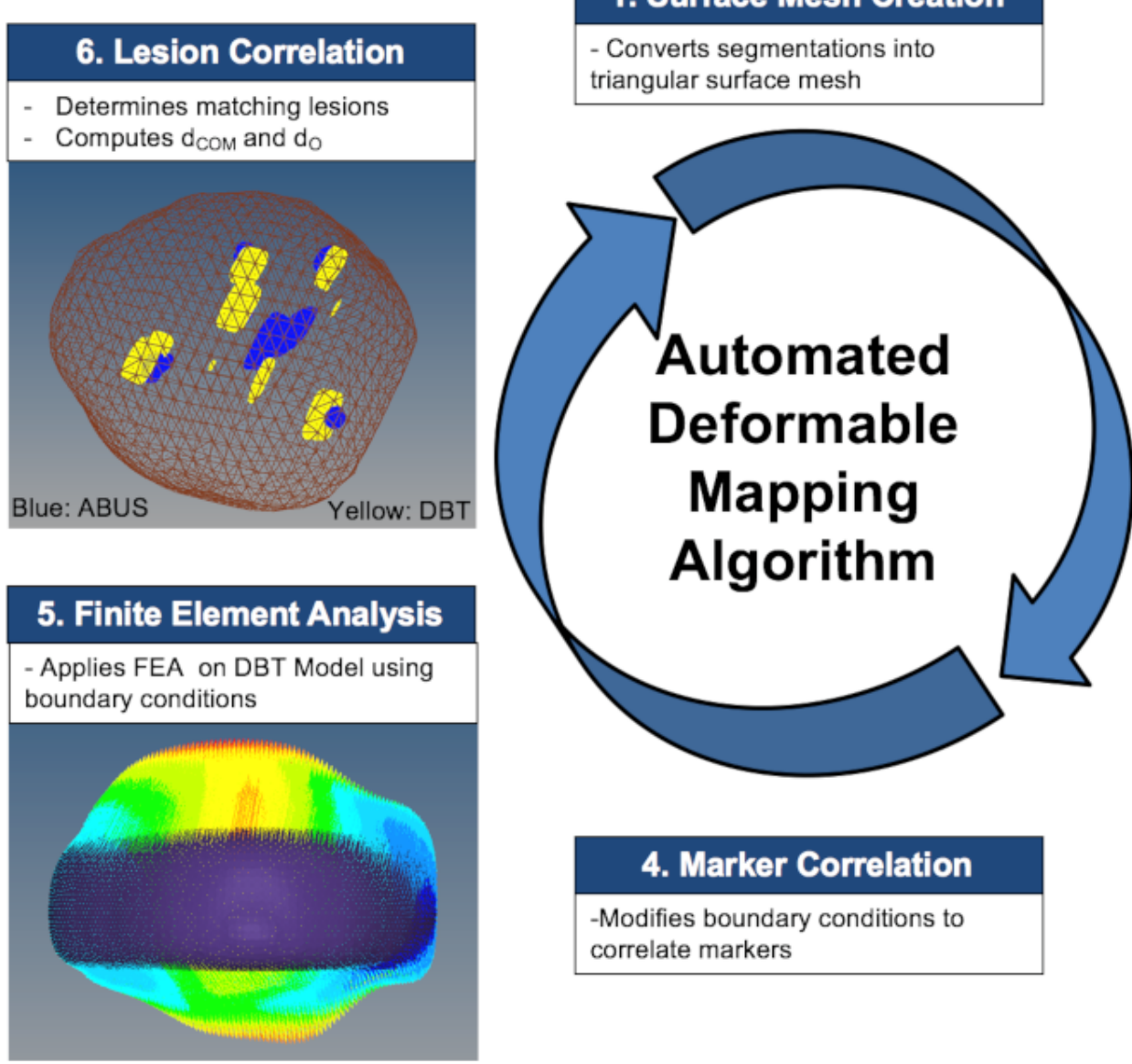

\section{Marker Correlation}

-Modifies boundary conditions to correlate markers
2. FE Model Generation

- Creates Base FE model

- Adds Material properties

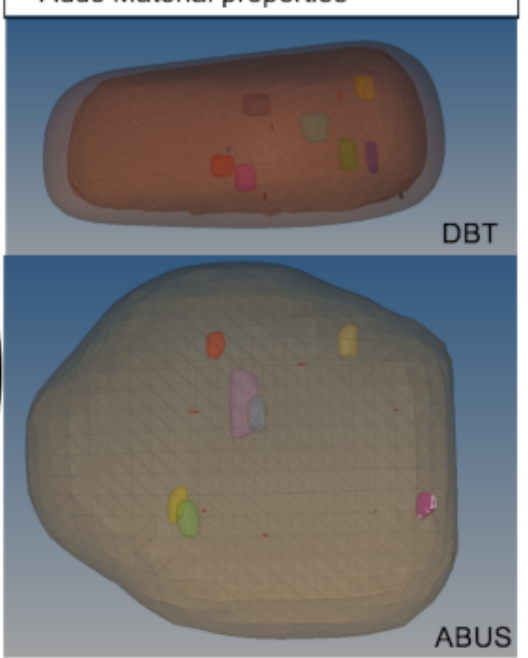

\section{Skin Deformation}

-Transforms DBT model to ABUS model

-Determines boundary conditions for FEA

FIG. 4. The automated deformable mapping algorithm process. [Color figure can be viewed at wileyonlinelibrary.com]

\section{E.2. Finite element model generation}

The algorithm uses the FE model pre-processing software, HyperMesh, to build the base FE model for the DBT and ABUS image set from the individual mesh triangular surface contours. 3D four-point tetrahedral FE models are created using the trias surface meshing algorithm within HyperMesh from all 3D-triangular surface mesh contours for each modality model, which results in fully connected tetrameshed base FE ABUS and DBT models. The algorithm takes into account the shape of the triangular surface being meshed and uses a defined library of element patterns to map them to triangular surfaces for tetrahedral mesh generation. Each reader's segmented dataset includes the resulting base DBT and ABUS models for both phantoms. The material properties that were assigned to the $3 \mathrm{D}$ tetrahedral model volumes are as noted in Table I. Surface interfaces are defined and boundary conditions are determined using a mesh morphing module named HyperMorph. The base DBT model is deformed to match the ABUS model since there is higher certainty in the overall breast shape in the DBT model. Since the simulated dense and cystic lesions cannot be distinguished in the DBT images, all lesions are assumed to have the material property of the dense lesions from Table I. For Phantom A, the average number of elements/nodes are $366,000 / 65,800$ for the base DBT model and 102,000/20,300 for the base ABUS model. For Phantom B the average number of nodes/ elements are 192,000/35,600 for the base DBT model and $137,000 / 24,200$ for the base ABUS model. The DBT model has a larger number of elements/nodes due to smaller element size since it is used for FEA and the ABUS model is used as the reference model.

\section{E.3. Skin surface transformation of DBT model to match ABUS skin surface}

The base DBT-FE model [Fig. 5(a), blue] is translated and rotated to the center of mass, COM, of the base ABUS FE model [Fig. 5(a), brown]. Nodal locations, (x, y, z-coordinate locations that define elements in FE model) from the skin surface of the base DBT-FE model are automatically moved to best match the outer surface of ABUS FE model along the axial and coronal planes by morphing the original mesh, which results in Fig. 5(c). This transformation reshapes the skin surface mesh and does not use any breast phantom material properties. For this transformation, the entire skin surface as the volume is encased in a six-sided hexahedron to encompass the entire skin volume. The handles, (68 in total, yellow and red spheres shown around the DBT skin mesh in Fig. 5) are created and encompass the outer contour of the DBT skin 
model at seven equidistant locations along each planar axis. The red spheres indicate global handles, which are eight in total and are generated at the eight corners of the hexahedron box surround the skin mesh. Global handles are used for making large scale changes to the mesh. The yellow spheres indicate local handles which make smaller scale changes to localized areas of the. There are 60 yellow spheres in total and are used to manipulate a small region of the skin mesh by influencing external nodal locations. Influence functions, using the HyperMorph feature within the HyperMesh software, relate the movement of the handles to the nodes within. There are a number of non-linear algorithms used depending on the size and shape of the domains and the number of nodes within. The algorithm enforces symmetry or constrains nodal movements in many different patterns to modify the relationship between handle movement and node manipulation. The external nodes of the DBT skin model are then related along the axial and coronal anatomical planes to the nearest node in the ABUS model based on the axes of interest. The external nodes of the DBT skin model are then related along the axial and coronal anatomical planes to the nearest node in the ABUS model based on the axes of interest.

The algorithm computes the differences and manipulates the handles so the DBT volume can match the external ABUS shape. Manipulating handles along the coronal plane [Fig. 5(b)], simulates the decompression of the DBT surface mesh. Manipulating handles along the axial plane [Fig. 5(c)], simulates AP compression of the DBT surface mesh to match the ABUS surface mesh. In other words, the original DBT surface mesh is morphed by changing nodal locations to better align with the overall shape of the ABUS surface mesh. The sagittal plane (the plane the ultrasound transducer is scanned) is not considered due to poor coupling along the right and left sides of the breast during ABUS image acquisition.

\section{E.4. External marker correlation}

After surface deformation is completed using the outer skin contours between models, the algorithm computes COM positions of the external fiducial markers from the morphed DBT model and reference ABUS model. The DBT surface mesh is further deformed based off external marker correlation as follows: External fiducial markers in the DBT model are matched with the base ABUS external fiducial markers by determining the minimum distance between the COMs of corresponding markers. This distance will be used to align markers closer together and allows for mesh corrections along all anatomical planes (includes correction along the scanning plane of the ultrasound transducer).

Once external markers are matched between the two sets and the resulting distances between COMs is determined, the algorithm determines which local handle [yellow spheres shown in Fig. 5(c)] is needed to adjust nodes within the mesh domain. This handle is determined by proximity to handle locations along the $x$-axis. Once the local handle is determined, the local handle is manipulated by half the distances between the COMs of correlated markers for each planar axis. This is repeated for all corresponding markers. After all handle manipulation is completed the algorithm recalculates the COM distances between markers. If all markers are within a user-defined distance, $\mathrm{d}_{\mathrm{M}}$ (between 1 and $10 \mathrm{~mm}$ ), the algorithm will begin FEA. If not, the algorithm identifies which markers are not within $d_{M}$ and iterates again to manipulate the handles for markers that are not satisfying the $\mathrm{d}_{\mathrm{M}}$ boundary condition. The algorithm will iterate until all markers satisfy the $d_{M}$ boundary condition. Each iteration will only perform handle corrections based off the total number of matched markers (i.e., six-matched markers corresponds to a maximum of six handle corrections for that iteration).

To maintain mesh integrity, the DBT-FE model is deformed by half the distance between correlated markers to ensure that mesh quality is not compromised due to large changes to the skin mesh which can halt processing by the FEA solver. Skin deformation per iteration occurs only for corresponding markers that are greater than $\mathrm{d}_{\mathrm{M}}$. For those markers, deformation by further manipulation of the handles, as described in Section 2.E.3., is performed in that area of the DBT model of the breast. A lower bound of $1 \mathrm{~mm}$ is used as it is approximately equal to the ultrasound point spread function expected for the fiducial markers. If there are no markers inferior to the nipple, mesh deformation is assumed symmetric superior and inferior to the nipple. The displacement for

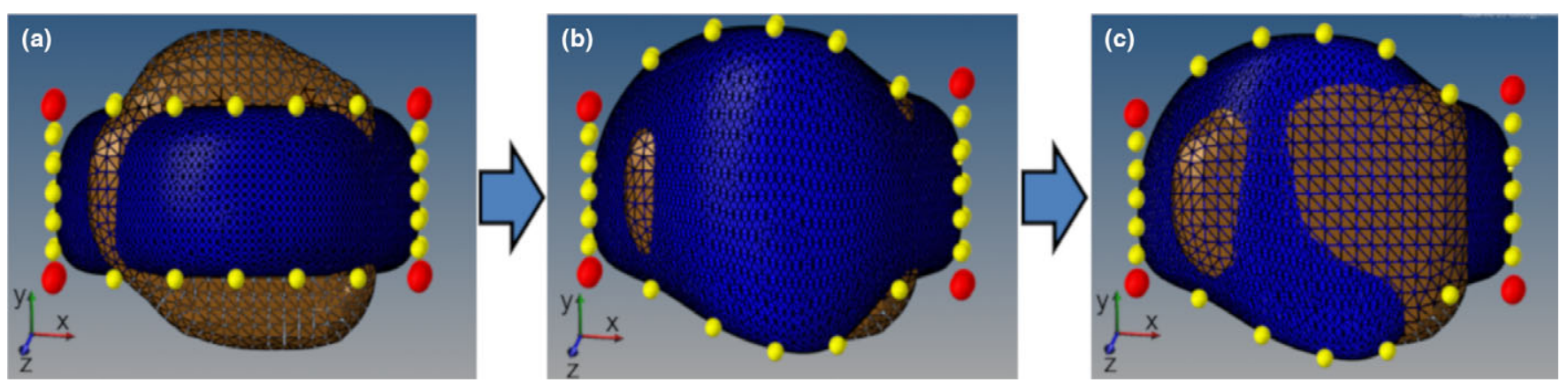

FIG. 5. Mesh transformation for external digital breast tomosynthesis (DBT) breast surface mesh to match shape of automated breast ultrasound (ABUS) surface mesh. Brown/Light: ABUS (unchanged) Blue/Dark: DBT (deformed) (a) COM translation and rotation of DBT to ABUS COM (b) Coronal handle manipulation of DBT breast surface mesh (along y axis) (c) Axial handle manipulation of DBT breast surface mesh (along $\mathrm{z}$ axis). [Color figure can be viewed at wileyonline library.com] 
each node from the surface mesh of the DBT translated model [Fig. 5(a)] and the deformed DBT surface mesh [Fig. 5(c) with the addition of external marker corrections] are stored and applied as boundary conditions for FEA deformation to begin on the base DBT model. Skin surface deformation is used for the sole purpose of creating the boundary conditions needed to begin the FEA.

\section{E.5. Finite element analysis}

The constraints or boundary conditions for each external DBT surface mesh are applied to the translated base DBT-FE model. These boundary conditions are used as input to the FEA solver program, Optistruct, to generate and solve the differential equations to describe the model deformation based off the defined constraints. The FEA uses the material properties and boundary conditions to find the resultant stress and strain of the deformation of the entire breast volume. Due to the high deformation that is needed to match the DBT and ABUS volumes, the FEA is performed as a non-linear quasi-static analysis in a single step. Linear static deformation would compromise mesh integrity and result in faulty output or inability of FEA to run. Therefore, this study does not recommend the use of linear static deformation analysis between DBT and ABUS compressions. The FEA was performed on a Windows 7 Intel ${ }^{\circledR}$ Core $^{\mathrm{TM}}$ i7 CPU speed of $2500 \mathrm{MHz}$ with 4 GB RAM with analysis complete in approximately $20 \mathrm{~min}$. The analysis time will be longer for models with greater complexity.

\section{E.6 Lesion correlation}

After FEA is performed, the COM of all lesions from the FEA-DBT model and the base FE ABUS model are determined. A correlation algorithm determines which lesions correspond to lesions in the other set with the constraint that the difference in COM is within $15 \mathrm{~mm}$. Previous studies, for deformable registration from breast MR to mammography $\mathrm{CC}$ views have shown mean registration errors of 10 $20 \mathrm{~mm} .{ }^{27}$ Therefore, since those studies were based on real breast data these bounds were used loosely as correlation criteria for registration measurement. All external nodal locations of each lesion are analyzed to determine overlap of correlated lesions. Corresponding lesions from DBT and ABUS sets that have a minimum distance between the COMs, $d_{\mathrm{COM}}$ are considered to match. For two lesions in DBT that have the same $d_{C O M}$ with a lesion in ABUS, the code checks the $\mathrm{d}_{\mathrm{COM}}$ of those two DBT lesions with other ABUS lesions to minimize all $\mathrm{d}_{\mathrm{COM}}$ and thereby determines the correct lesion matches. The matching process is described in pseudo-code in the Data $\mathrm{S} 1$ for this paper.

All corresponding lesions are considered matched between the two modality sets based on the criteria in Table II. Since our studies use uniform background phantoms with large numbers of lesions, stricter lesion correlation guidelines were employed to reduce the likelihood of mismatch.

Therefore, if two corresponding lesions overlap they are considered a match if $\mathrm{d}_{\mathrm{COM}}$ is within $10 \mathrm{~mm}$. If lesions
TABLE II. Criteria for lesion correlation between automated breast ultrasound (ABUS) and digital breast tomosynthesis (DBT) models.

\begin{tabular}{lll}
\hline \hline & \multicolumn{1}{c}{$\mathrm{d}_{\mathrm{COM}}$} & \multicolumn{1}{c}{$\mathrm{d}_{\mathrm{O}}$} \\
\hline Overlapping lesions & $\mathrm{d}_{\mathrm{COM}} \leq 10 \mathrm{~mm}$ & $\mathrm{n} / \mathrm{a}$ \\
Non-overlapping lesions & $\mathrm{d}_{\mathrm{COM}} \leq 15 \mathrm{~mm}$ & $\mathrm{~d}_{\mathrm{O}} \leq 7.5 \mathrm{~mm}$ \\
\hline \hline
\end{tabular}

correspond but do not overlap the minimum distance to overlap, $\mathrm{d}_{\mathrm{O}}$, is calculated as shown in Fig. 6. If the two corresponding lesions are within a $\mathrm{d}_{\mathrm{COM}}$ of $15 \mathrm{~mm}$ and $\mathrm{d}_{\mathrm{O}}$ is within $7.5 \mathrm{~mm}$, the lesions are matched. The $\mathrm{d}_{\mathrm{O}}$ is restricted to $7.5 \mathrm{~mm}$ to ensure a match discrepancy is not made. The results are output into a table and can be used to relate positions from the original ABUS and DBT DICOM data. Calcifications from Phantom A are not considered during lesion matching.

\section{F. Studies performed}

The following studies were performed:

- Inter-reader concordance of segmented lesions: Interreader concordance of all segmented lesions was evaluated using DSC and distances between the COM's of corresponding lesions.

- Inter-reader concordance of locations of fiducial markers: Inter-reader concordance of the locations of the fiducial markers was evaluated by measuring the distances between the COM's of each segmented marker for each image set.

- Reader reproducibility: After segmentation was performed for all lesions, three lesions were selected from each image set of each phantom for a study of reader segmentation reproducibility. Each reader segmented those three lesions in the DBT and ABUS images several days after completing their original segmentations.

- Accuracy of deformable mapping technique: For Phantom A, results were compared for cases when sufficient iterations were performed to achieve corresponding marker separations of $\leq 1 \mathrm{~mm}$ vs $\leq 5 \mathrm{~mm}$. This comparison included results when different numbers of markers were used. In addition, results were compared with and without the use of the markers. For all comparisons, the statistical significance of any differences between the average $d_{\text {COM }}$ values for corresponding lesions were determined with paired t-tests. The numbers of lesions that overlapped and $\mathrm{d}_{\mathrm{O}}$ were also compared.

\section{RESULTS}

\section{A. Analysis of inter-reader concordance of segmented lesions in both phantoms}

Table III compares the segmentation results between readers for all lesions in both phantoms. The DSC for the ABUS data for both phantoms are approximately 0.90. For DBT 

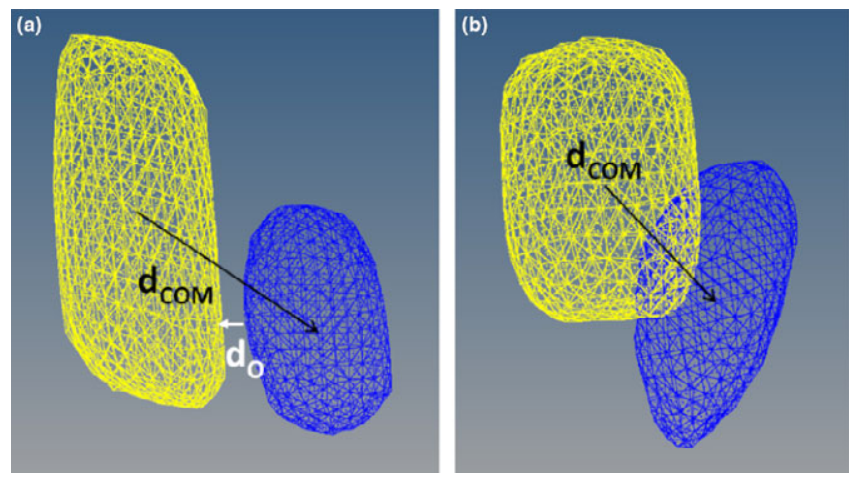

FIG. 6. Lesion correlation metrics for COM distance $\left(\mathrm{d}_{\mathrm{COM})}\right.$ and minimum distance to overlap $\left(\mathrm{d}_{\mathrm{O}}\right)$ for (a) non-overlapping lesions and (b) overlapping lesions. $($ Blue/Dark $=$ ABUS, Yellow/Light $=\mathrm{DBT})$. $[$ Color figure can be viewed at wileyonlinelibrary.com]

data, for both phantoms, DSC results are lower, likely due to differences in reader determination of the vertical extents of the lesions. The smallest DSC is 0.57 for R2 to R3 for Phantom 2. For this same reader correlation, the average distance between COM $\left(\mathrm{d}_{\mathrm{COM}}\right)$ is $0.81 \mathrm{~mm}$ thus illustrating that even though the DSC values are low, the central positions of the lesions segmented by the different readers are still very close to one another.

\section{B. Analysis of inter-reader concordance of fiducial markers locations}

Table IV illustrates the average distances between the COM's of the fiducial markers segmented by the three readers. The largest differences between the COM's for both DBT and ABUS are approximately $2 \mathrm{~mm}$.

\section{C. Reader reproducibility analysis}

Table V illustrates the reproducibility of the segmentation results for each individual reader for three lesions in Phantom A and Phantom B. The reproducibilities of the DSC for the individual readers are in general higher for the lesions in the ABUS images and lower in the DBT images. The latter is likely due to differences in reader determination of the vertical extents of the lesions in DBT images when the readers repeat the segmentations themselves. However, the $\mathrm{d}_{\mathrm{COM}}$ values in
Table $\mathrm{V}$ are less than $2 \mathrm{~mm}$ for both modality images indicating good reproducibility of the positions of the lesions.

For segmentations by each reader, deformation was successfully performed on the base DBT-FE model and correlated with the corresponding ABUS datasets between reader segmented data. In every iteration of the algorithm, the average distances between the COMs between the readers of the correlated markers decreased as shown in Fig. 6. After six iterations, the average correlated difference between all markers is $3.0 \mathrm{~mm}$ which corresponds to $\mathrm{a} \mathrm{d}_{\mathrm{M}} \leq 5 \mathrm{~mm}$.

\section{D. Accuracy of deformable mapping for different numbers of fiducial makers for phantom $A$}

Phantom A has seven lesions, all of which were viewed with ABUS. However, only six of the lesions were viewed in the DBT images because one lesion was too close to the chest wall and moved outside the image field of view when the phantom was compressed. Figure 7(a), illustrates the resulting lesion $\mathrm{d}_{\mathrm{COM}}, \mathrm{d}_{\mathrm{O}}$, and overall lesion overlap where no marker analysis was used and compares it to various marker combinations described in Section 2.B when markers within a 1-mm distance $\left(d_{M} \leq 1 \mathrm{~mm}\right)$. Figure $7(b)$, illustrates the resulting lesion $\mathrm{d}_{\mathrm{COM}}, \mathrm{d}_{\mathrm{O}}$, and overall lesion overlap fraction that were obtained when no fiducial marker analysis was used and compares it to various marker combinations described in Section 2.B when all corresponding markers were within a 5 - $\mathrm{mm}$ distance $\left(\mathrm{d}_{\mathrm{M}} \leq 5 \mathrm{~mm}\right)$.

For the six marker case, six iterations were needed for all of the corresponding external markers to be within a 5-mm distance of each other. For all corresponding external markers to

TABLE IV. Average COM distance $\left(\mathrm{d}_{\mathrm{COM}}\right)$ between external markers among readers in digital breast tomosynthesis (DBT) and automated breast ultrasound (ABUS) data sets for Phantoms A and B.

\begin{tabular}{lcccc}
\hline \hline & \multicolumn{4}{c}{$\mathrm{d}_{\mathrm{COM}}(\mathrm{mm})$} \\
\cline { 2 - 4 } & \multicolumn{2}{c}{ Phantom A } & & \multicolumn{2}{c}{ Phantom B } \\
\cline { 2 - 3 } \cline { 5 - 6 } & ABUS & DBT & ABUS & DBT \\
\hline R1 to R2 & $1.31 \pm 0.37$ & $1.37 \pm 0.26$ & $0.45 \pm 0.27$ & $1.18 \pm 0.26$ \\
R2 to R3 & $1.33 \pm 0.40$ & $0.69 \pm 0.08$ & $0.74 \pm 0.23$ & $1.38 \pm 0.34$ \\
R3 to R1 & $1.77 \pm 0.61$ & $0.97 \pm 0.32$ & $0.48 \pm 0.27$ & $2.24 \pm 0.72$ \\
\hline \hline
\end{tabular}

TABLE III. Average DSC and average COM distance $\left(\mathrm{d}_{\mathrm{COM}}\right)$ results between readers for corresponding lesions in all digital breast tomosynthesis (DBT) and automated breast ultrasound (ABUS) data sets for Phantoms A and B.

\begin{tabular}{|c|c|c|c|c|c|c|c|c|}
\hline & \multicolumn{2}{|c|}{ ABUS } & \multicolumn{2}{|c|}{ DBT } & \multicolumn{2}{|c|}{ ABUS } & \multicolumn{2}{|c|}{ DBT } \\
\hline $\mathrm{R} 1$ to $\mathrm{R} 2$ & $0.89 \pm 0.07$ & $0.20 \pm 0.03$ & $0.70 \pm 0.19$ & $1.06 \pm 0.27$ & $0.90 \pm 0.05$ & $0.31 \pm 0.06$ & $0.62 \pm 0.24$ & $0.78 \pm 0.08$ \\
\hline $\mathrm{R} 2$ to $\mathrm{R} 3$ & $0.88 \pm 0.08$ & $0.29 \pm 0.01$ & $0.73 \pm 0.11$ & $0.59 \pm 0.12$ & $0.88 \pm 0.09$ & $0.30 \pm 0.05$ & $0.57 \pm 0.25$ & $0.81 \pm 0.17$ \\
\hline
\end{tabular}


TABLE V. Reproducibility for each reader's lesion segmentations in digital breast tomosynthesis (DBT) and automated breast ultrasound (ABUS) data sets for Phantoms A and B using DSC coefficients and average COM distance $\left(\mathrm{d}_{\mathrm{COM}}\right)$.

\begin{tabular}{|c|c|c|c|c|c|c|c|c|}
\hline & \multicolumn{2}{|c|}{ ABUS } & \multicolumn{2}{|c|}{ DBT } & \multicolumn{2}{|c|}{ ABUS } & \multicolumn{2}{|c|}{ DBT } \\
\hline $\mathrm{R} 1$ & $0.85 \pm 0.04$ & $0.50 \pm 0.27$ & $0.78 \pm 0.09$ & $1.88 \pm 1.34$ & $0.89 \pm 0.02$ & $0.19 \pm 0.03$ & $0.68 \pm 0.19$ & $0.59 \pm 0.05$ \\
\hline $\mathrm{R} 2$ & $0.86 \pm 0.03$ & $0.47 \pm 0.34$ & $0.85 \pm 0.05$ & $0.60 \pm 0.45$ & $0.86 \pm 0.01$ & $0.23 \pm 0.13$ & $0.66 \pm 0.16$ & $0.69 \pm 0.05$ \\
\hline
\end{tabular}

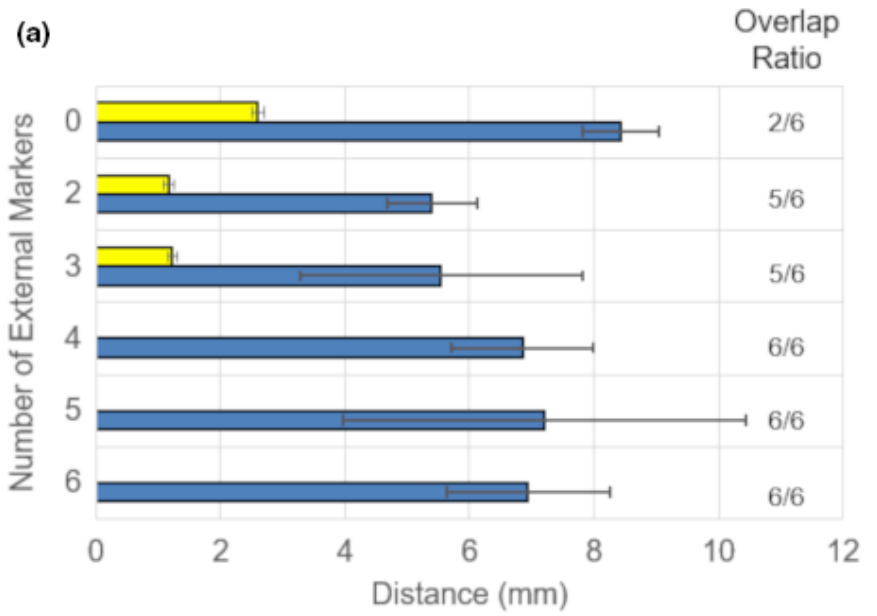

$\square$ Minimum Distance to Overlap $\quad$ COM Distance

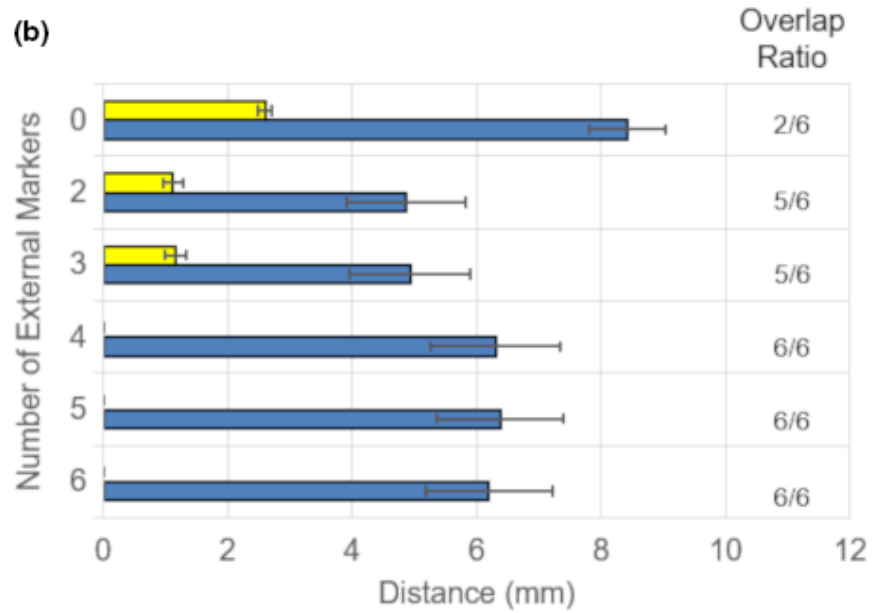

$\square$ Minimum Distance to Overlap $\quad$ cOM Distance

FIG. 7. Average distances between COMs of corresponding lesions in automated breast ultrasound (ABUS) and digital breast tomosynthesis (DBT) images $\left(\mathrm{d}_{\mathrm{COM}}\right)$ for all 3 readers, as well as minimum distance to overlap $\left(\mathrm{d}_{\mathrm{O}}\right)$, and overall lesion overlap ratio (the number of lesions that overlapped between DBT and ABUS sets divided by the total number of lesions that were imaged) for Phantom A with various number of external fiducial markers. (a) All marker distances were within a distance $\left(\mathrm{d}_{\mathrm{M}} \leq 1 \mathrm{~mm}\right)$ between readers' data sets. (b) All marker distances were within a distance ( $\left.\mathrm{d}_{M} \leq 5 \mathrm{~mm}\right)$ between readers' data sets. Marker combinations from Fig. 1:6 markers (A-F), five Markers (A, B, C, E, and F), four markers (F, B, E, and C), three markers (A, B, and F) and two markers (F and B). [Color figure can be viewed at wileyonlinelibrary.com]

be within a 1-mm distance of each other required 17 iterations. Each iteration can have runtimes of up to 3 min depending on the number of markers used in the analysis. Therefore, a difference of 10 iterations can increase runtime by about $30 \mathrm{~min}$. Although as shown in Fig. 7, the $\mathrm{d}_{\mathrm{COM}}$ are slightly greater for markers being within $1 \mathrm{~mm}$ vs $5 \mathrm{~mm}$ (likely due to the greater number of iterations and corrections made to get all of the markers within the $1 \mathrm{~mm}$ distance), the $P$-value for a paired $t$ test was 0.12 . Thus, there was no statistical difference in $\mathrm{d}_{\mathrm{COM}}$ when markers were within $1 \mathrm{~mm}$ vs a 5 -mm distance. Hence, a $\mathrm{d}_{\mathrm{M}}$ of $5 \mathrm{~mm}$ is regarded as an acceptable distance between correlated markers to ensure acceptable algorithm run time, with the employed, simple processor, while maintaining desired lesion correlation. Without the use of markers only two of six lesions overlapped. For both $\mathrm{d}_{\mathrm{M}}$ cases analyzed, overlap improved to six of six lesions with 4-6 marker analysis combinations and improved to five of six lesions overlapped for two and three marker analyses. The largest improvement in $\mathrm{d}_{\mathrm{COM}}$ is shown for two and three markers vs zero markers. Figure 8 displays the improvement from no marker analysis to that with three marker analyses in Phantom A for all correlated lesions for Reader one segmented datasets.
Table VI, compares the average distances between the COMs of corresponding individual lesions \pm the standard deviations of those distances in the deformed DBT and base ABUS images for the combined data for all three readers for phantom A, without the use of markers and with the use of three external markers for lesion correlation. The mean difference in lesion $\mathrm{d}_{\mathrm{COM}}$ was $1.9 \mathrm{~mm}$ with three marker analyses vs without. A paired $t$-test of the mean $\mathrm{d}_{\mathrm{COM}}$ values was performed and resulted with an overall $P$-value of 0.01 for the averaged dataset. Therefore, the use of three external markers showed statistically significant improvements in lesion $\mathrm{d}_{\mathrm{COM}}$ in comparison to the use of no external markers.

\section{E. Accuracy of deformable mapping technique for phantom B}

Based on the results for Phantom A, Phantom B was only imaged with three markers in the positions $\mathrm{F}, \mathrm{A}$, and $\mathrm{B}$ as illustrated in Fig. 1. Phantom B has 12 lesions, all of which were viewed with ABUS. However, only seven of the lesions were viewed in the DBT images, because five lesions were too close to the chest wall and moved outside the image field 

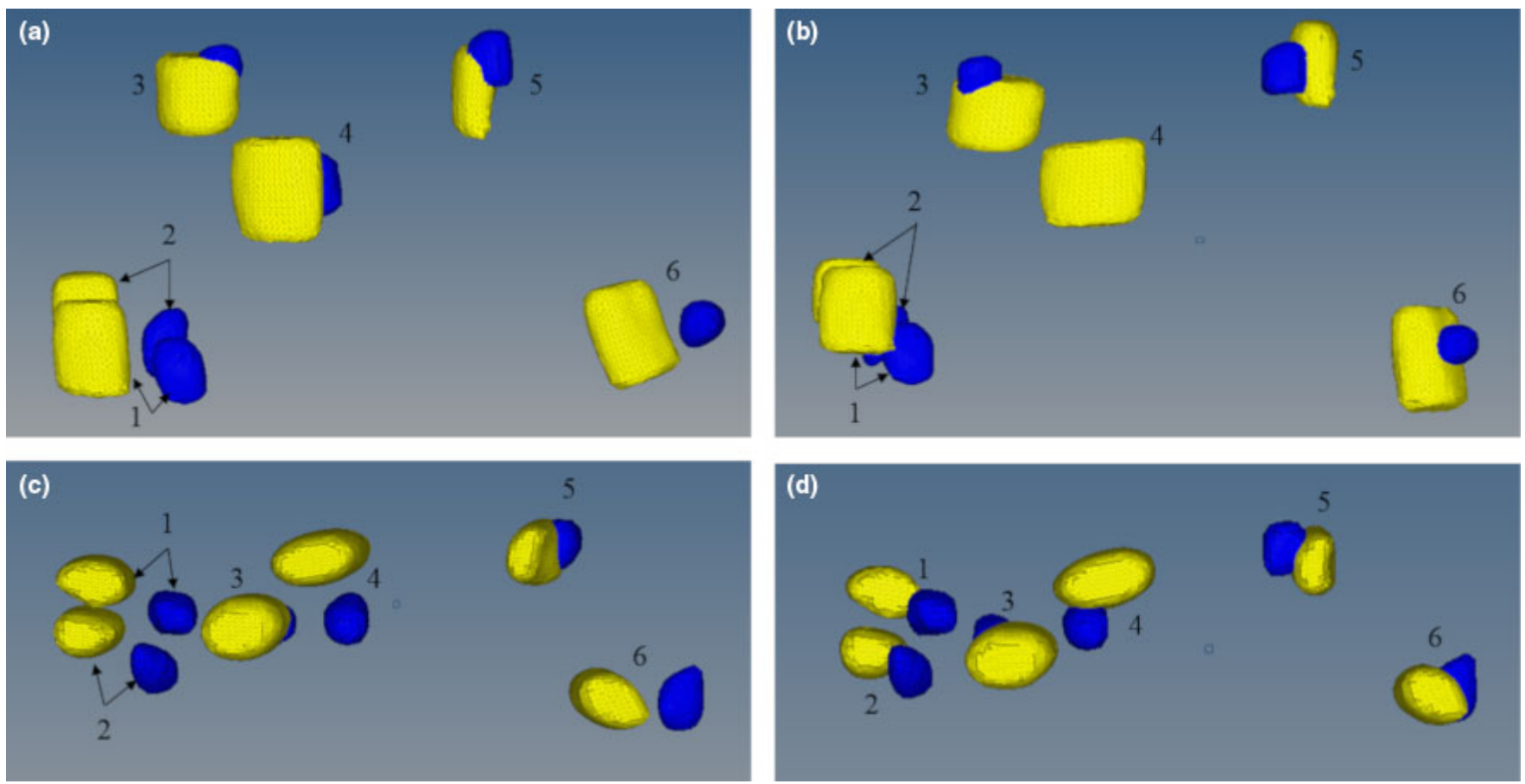

FIG. 8. Phantom A lesion correlation for Reader 1 (a) without marker analysis coronal view (b) with marker analysis coronal view (c) without marker analysis axial view (d) with marker analysis axial view. All numeric values correspond with Lesion ID's in Table VI. (Blue/Dark $=$ ABUS, Yellow/Light $=$ DBT). [Color figure can be viewed at wileyonlinelibrary.com]

\begin{tabular}{|c|c|c|c|c|c|c|c|}
\hline \multicolumn{4}{|c|}{ Without marker analysis } & \multicolumn{4}{|c|}{ With 3 marker analysis $\left(\mathrm{d}_{\mathrm{M}} \leq 0.5 \mathrm{~mm}\right)$} \\
\hline Lesion ID & $\mathrm{d}_{\mathrm{COM}}(\mathrm{mm})$ & $\mathrm{d}_{\mathrm{O}}(\mathrm{mm})$ & Overlap & Lesion ID & $\mathrm{d}_{\mathrm{COM}}(\mathrm{mm})$ & $\mathrm{d}_{\mathrm{O}}(\mathrm{mm})$ & Overlap \\
\hline 1 & $8.2 \pm 0.3$ & $3.4 \pm 0.4$ & No & 1 & $4.5 \pm 0.2$ & $\mathrm{n} / \mathrm{a}$ & Yes \\
\hline 2 & $10.2 \pm 0.7$ & $3.6 \pm 0.3$ & No & 2 & $7.1 \pm 1.1$ & $\mathrm{n} / \mathrm{a}$ & Yes \\
\hline 3 & $5.0 \pm 0.3$ & $\mathrm{n} / \mathrm{a}$ & Yes & 3 & $3.9 \pm 0.5$ & $\mathrm{n} / \mathrm{a}$ & Yes \\
\hline 4 & $8.5 \pm 0.1$ & $1.9 \pm 0.1$ & No & 4 & $8.4 \pm 0.5$ & $1.1 \pm 0.0$ & No \\
\hline 5 & $4.7 \pm 0.5$ & $\mathrm{n} / \mathrm{a}$ & Yes & 5 & $2.0 \pm 0.5$ & $\mathrm{n} / \mathrm{a}$ & Yes \\
\hline 6 & $4.5 \pm 0.1$ & $1.5 \pm 0.6$ & No & 6 & $3.6 \pm 1.4$ & $\mathrm{n} / \mathrm{a}$ & Yes \\
\hline Average & 6.8 & 2.6 & Overlap & Average & 4.9 & 1.1 & Overlap \\
\hline$\sigma$ & 2.4 & 1.1 & $2 / 6$ & $\sigma$ & 2.4 & 0.0 & $5 / 6$ \\
\hline
\end{tabular}

TABLE VI. Lesion correlation for Phantom A when no external markers are used for the deformation (left) vs three external markers used for the deformation (right).

of view when the phantom was compressed. Without the use of markers analysis, four lesions were correlated (within $15 \mathrm{~mm}$ of each other) with only one overlapped. As shown in Table VII, the average $\mathrm{d}_{\mathrm{COM}}$ between corresponding ABUS and DBT lesions for the three readers was $9.7 \pm 3.5 \mathrm{~mm}$ and the average lesion $\mathrm{d}_{\mathrm{O}}$ was $2.9 \pm 1.8 \mathrm{~mm}$. For the segmented data without marker analysis only one lesion showed overlap. These results are tabulated in Table VII.

Table VII, also includes a comparison between the deformable mapping method without marker analysis with that for two and three external markers. Using two and three marker analyses, the average $\mathrm{d}_{\mathrm{COM}}$ between corresponding lesions improved by $18 \%$ and $27 \%$, respectively, relative to the no markers case. For the four lesions correlated without marker analysis a paired $t$-test of the mean $\mathrm{d}_{\mathrm{COM}}$ values was performed with the results for two and three marker analysis combinations and both resulted in $P$-values of 0.01 respectively.
Therefore, these four lesions on average show statistically significant improvement in lesion $\mathrm{d}_{\mathrm{COM}}$ with the use of external markers. Figure 9 displays the improvement from no marker analysis to that with three marker analyses in Phantom B for all correlated lesions for Reader one segmented datasets.

Table VII, shows a comparison between the deformable mapping method without marker analysis and with two and three external markers. The mean $\mathrm{d}_{\mathrm{COM}}$ values for two and three marker analyses are $7.6 \pm 3.6 \mathrm{~mm}$ and $8.5 \pm 4.0 \mathrm{~mm}$, respectively. A paired two-sample $t$-test for means was performed on the average $\mathrm{d}_{\mathrm{COM}}$ values. The $P$ value was 0.053 and supports that the difference between using two markers and three markers is not statistically significant. Lesion ID $=7$ values were calculated for only two readers, as that specific lesion was not within bounds for correlation for one of the readers as defined in Table II. 
TABLE VII. Lesion correlation for Phantom B when no external markers are used for the deformation and with the use of three and two marker analysis. Note: Lesion Overlap is defined by the resultant of at least two of three readers' data showing overlap for that specific lesion between automated breast ultrasound (ABUS) and digital breast tomosynthesis (DBT) set. ${ }^{+}$Indicates that lesion 3 in no marker analysis showed overlap for two of three readers' datasets. *Indicates that lesion 7 was out of correlation bounds for one reader set and therefore the values are averaged based for two readers' data and not all three.

\begin{tabular}{|c|c|c|c|c|c|c|c|c|c|c|c|}
\hline \multicolumn{4}{|c|}{ Without marker analysis } & \multicolumn{4}{|c|}{ With two marker analysis $\left(\mathrm{d}_{\mathrm{M}} \leq 0.5 \mathrm{~mm}\right)$} & \multicolumn{4}{|c|}{ With three marker analysis $\left(\mathrm{d}_{\mathrm{M}} \leq 0.5 \mathrm{~mm}\right)$} \\
\hline Lesion ID & $\mathrm{d}_{\mathrm{COM}}(\mathrm{mm})$ & $\mathrm{d}_{\mathrm{O}}(\mathrm{mm})$ & Overlap & Lesion ID & $\mathrm{d}_{\mathrm{COM}}(\mathrm{mm})$ & $\mathrm{d}_{\mathrm{O}}(\mathrm{mm})$ & Overlap & Lesion ID & $\mathrm{d}_{\mathrm{COM}}(\mathrm{mm})$ & $\mathrm{d}_{\mathrm{O}}(\mathrm{mm})$ & Overlap \\
\hline 2 & $8.1 \pm 0.5$ & $2.9 \pm 0.4$ & No & 2 & $3.4 \pm 0.9$ & $\mathrm{n} / \mathrm{a}$ & Yes & 2 & $2.9 \pm 1.5$ & $\mathrm{n} / \mathrm{a}$ & Yes \\
\hline $3^{+}$ & $5.8 \pm 2.4$ & $0.8 \pm 0.0$ & Yes & 3 & $3.4 \pm 0.7$ & $\mathrm{n} / \mathrm{a}$ & Yes & 3 & $3.3 \pm 2.0$ & $\mathrm{n} / \mathrm{a}$ & Yes \\
\hline 4 & $11.1 \pm 2.0$ & $5.2 \pm 1.3$ & No & 4 & $6.7 \pm 1.5$ & $\mathrm{n} / \mathrm{a}$ & Yes & 4 & $8.3 \pm 1.6$ & $1.1 \pm 1.3$ & No \\
\hline 6 & $\mathrm{n} / \mathrm{a}$ & $\mathrm{n} / \mathrm{a}$ & $\mathrm{n} / \mathrm{a}$ & 6 & $11.1 \pm 2.7$ & $4.0 \pm 0.4$ & No & 6 & $11.8 \pm 3.7$ & $5.1 \pm 1.6$ & No \\
\hline 7 & $\mathrm{n} / \mathrm{a}$ & $\mathrm{n} / \mathrm{a}$ & $\mathrm{n} / \mathrm{a}$ & $7 *$ & $12.7 \pm 0.7$ & $5.8 \pm 0.1$ & No & $7 *$ & $12.8 \pm 0.2$ & $6.6 \pm 0.8$ & No \\
\hline Average & 9.7 & 2.9 & Overlap & Average & 7.6 & 3.6 & Overlap & Average & 8.5 & 3.6 & Overlap \\
\hline$\sigma$ & 3.5 & 1.8 & $1 / 4$ & $\sigma$ & 3.6 & 2.5 & $4 / 7$ & $\sigma$ & 4 & 2.6 & $3 / 7$ \\
\hline
\end{tabular}
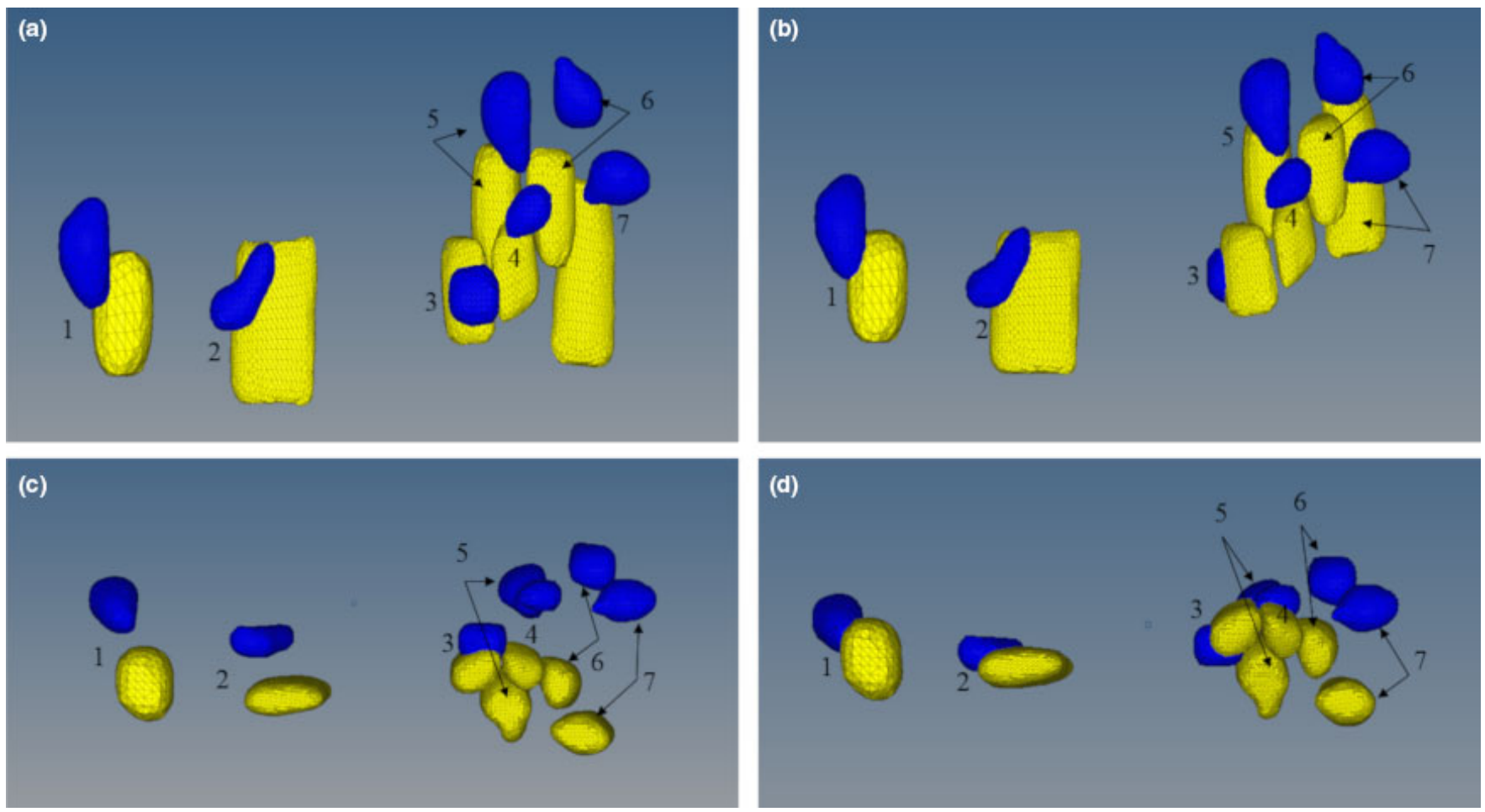

FIG. 9. Phantom B lesion correlation for Reader 1 (a) without marker analysis in the coronial view (b) with three marker analyses in the coronial view (c) without marker analysis in the axial view (d) with three marker analyses in the axial view. All numeric values correspond with Lesion ID's in Table IV. Note that for the without marker analysis lesions five, six, and seven did not meet the correlation criteria of being within $15 \mathrm{~mm}$ of each other (See Table VII). (Blue/ Dark $=$ ABUS, Yellow/Light $=$ DBT). [Color figure can be viewed at wileyonlinelibrary.com]

\section{DISCUSSION}

A novel automated deformable mapping algorithm has been described and assessed between upright DBT and supine ABUS images. Although this method uses commercially available biomechanical modeling and FEA software, the techniques described can be applied to other commercially available software or research algorithms. This study demonstrates that with the use of two and three external fiducial markers for the deformation results in up to $28 \%$ improvement in lesion correlation $\left(\mathrm{d}_{\mathrm{COM}}\right)$ in comparison with not using external markers. An expansion of this work, will incorporate an interface on a radiologist work station for displaying corresponding lesions in the original ABUS and DBT slices as shown Fig. 10.

Figure 7 demonstrates that for Phantom A, as the number of external fiducial markers that are used for deformable mapping increase from 4 to 6 , the overlap fraction remains the same and the average $\mathrm{d}_{\mathrm{COM}}$ remains approximately the same. This effect is probably a result of Phantom A being a stiff 


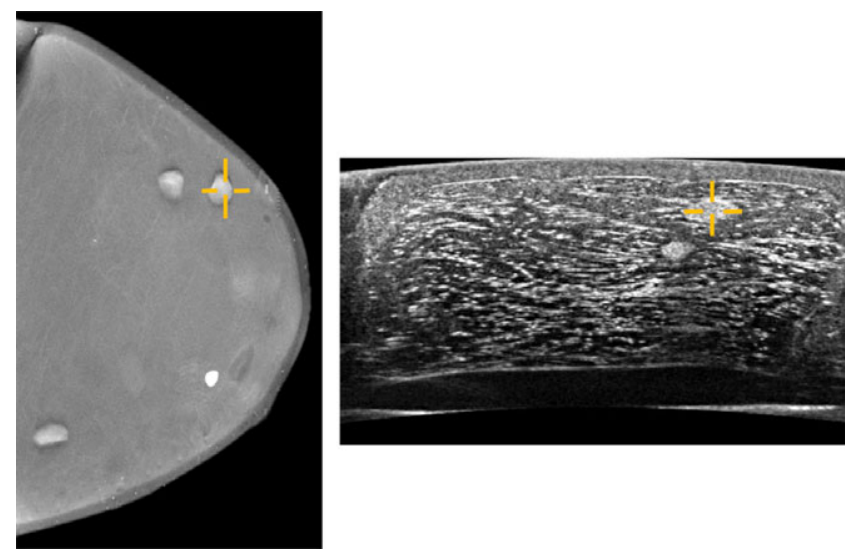

FIG. 10. Relating corresponding lesion in digital breast tomosynthesis (DBT) and automated breast ultrasound (ABUS) original datasets for Phantom A based on use of deformable mapping algorithm results. [Color figure can be viewed at wileyonlinelibrary.com]

phantom. It should be noted that although, in this study, two different compression forces were used, and the phantoms represented two different breast densities, the automated deformable mapping algorithm was successful in identifying the majority of the corresponding lesions within the two phantom data sets. This supports translation of the deformable mapping method to actual patient data, where there will be a wide range of compressions used as well as differences in breast density and size.

For Phantom B, without marker analysis only four lesions were correlated. Comparative $t$-tests of the means of the $\mathrm{d}_{\mathrm{COM}}$ of those lesions without marker analysis to those with two and three marker combinations indicated statistical significance. With the use of two and three markers all seven lesions came within correlation parameters. However, a statistically insignificant $t$-test of $\mathrm{d}_{\mathrm{COM}}$ between the use of two and three markers indicates no greater improvement in correlation with the use of three markers over two in the indicated geometry. Nevertheless, additional markers in the axillary region, which was not simulated in the phantoms for this study could potentially allow better registration in patient imaging. This will be investigated in future studies.

It is difficult to directly compare the results between Phantom A and Phantom B for the following reasons: Phantom A used more external markers than Phantom B. Phantom B did not contain the Z-skin membrane and all lesions were near the chest wall. Phantom B was easily compressed simulating a fatty breast and Phantom A represented a breast with higher glandular tissue content. Using only three external markers for Phantom B vs six markers for phantom A may have biased the results. Using the same number of external markers for both phantoms would have allowed for a better direct comparison between the two data sets.

Overall, the phantoms were easily segmented by readers. The largest differences between readers and variance in reader reproducibly were evident in the DBT data sets. Due to poor axial resolution in DBT imaging, determining where lesions were blurred or out of focus at the superior and inferior extents of the lesions was difficult. Therefore, the use of an automated segmentation method would be useful and will be investigated for future work. Drawing the top and bottom of the lesions at a distance from the central plane equal to the mean circumference in the central plane might prove most effective. After lesions are correlated, their borders in DBT might be drawn better based on the ABUS contours.

When deforming the DBT model to the base ABUS model this study assumes that all lesions are homogenous and have the material properties of dense lesions. After correlation has taken place, for lesions that correlated with cystic lesions in the ABUS set the algorithm could be rerun with the correct cystic material properties. In actual patient data, there will be heterogeneity in breast tissue. An expansion of this study could investigate the significance of this heterogeneity in the FEA-based registration.

The use of two or three markers for deformable mapping with Phantom A showed improvements for $\mathrm{d}_{\mathrm{COM}}$ results over the use of 4-6 markers. Based on marker placement in this study, markers located inferior to the nipple have direct contact with the breast support plate during DBT compression. The inferior part of the phantom flattens initially when the phantom is placed in contact with the breast support plate. It is believed that this contact restricts the movement of the markers resulting in small displacements of the markers in this region. The contact could also cause the markers to stick to the breast support plate during compression and fall off the breast eliminating their use in the subsequent ABUS scans and therefore in the deformable registration.

Conversely, markers located superior to the nipple (Fig. 1. Positions A, B, and F) did not have initial contact with the DBT compression paddle and the marker displacements were not as easily compromised between DBT and ABUS imaging. Results from both phantoms indicate that a minimum of two or three external markers at the indicated positions provide significant improvement in lesion registration. However, we intend to directly quantitate the number of markers and their respective locations that are the most helpful in an IRB approved proof-of-concept study with patients. Likewise, an MRI to US breast study also used three external markers at those approximate locations and found improvement in lesion registration. $^{35}$

Intuitively, one would suspect that more external markers would allow for better correlation. However, this study indicates that when modeling large breast compressions, external deformation may not be completely indicative of internal breast deformation. The degree to which this holds true for real breasts is yet to be determined. We believe that determination of optimal external marker positions on the breast could yield better results in lesion correlation, which could allow the use of fewer markers. A recent study using external fiducial markers to register MR breast images with microwave images found that fewer better placed fiducial markers reduced the effect of skin translation that is associated with movement of internal structures in the breast. ${ }^{46}$

The multimodality phantoms used in this work have limitations with respect to simulating real breasts. Some of these limitations include: (a) they are made of materials that follow 
a linear elastic behavior while under compression; whereas, real breast tissue follows hyperelastic behavior, ${ }^{20,38,39}$ (b) they have a homogeneous background; whereas, real breasts have a heterogeneous background and (c) they do not simulate attachment to the pectoral structure which could also contribute to the linear elastic behavior. Our planned human subject study will determine the degree to which these limitations affect the results in patients. To reduce these effects, we will use automatic segmentation of the DBT images into glandular and adipose tissues and include the elasticity properties of those tissues in our model. Further developments are needed to produce physical breast phantoms with materials that better model patient-specific breast tissue properties and can model the axillary region of the breast. Other deformable breast registration studies have proposed the use of patientspecific in vivo parameters to determine biomechanical properties. ${ }^{21,23}$

During ABUS imaging, up to three scans are rendered to image the breast in its entirety. The Invenia system scans from the superior to inferior margins of the breast producing axial images. The scans are performed in an AP view. For different views of the breast, this process can be repeated with parasagittal imaging planes. Multiple ABUS scans allow the ability to estimate the patient-specific distribution of elastic properties in the breast. Inclusion of ABUS-based elastography or pulse echo segmentation ${ }^{47}$ could offer great benefits for the deformable mapping algorithm and other biomechanical techniques for use in FEA. With more ABUS scans, the need for an additional marker near the axilla region of the breast could be helpful in relating ABUS projections. This study only considers one ABUS volume; whereas, up to three volumes can be taken during an ABUS exam of a patient. Stitching or overlaying ABUS views into one volume would be helpful and provide better coverage for deformation of the DBT-FE base algorithm. ABUS stitching or overlay could also allow this technique to deform the base ABUS model into the DBT base model. This would allow the correct cystic and dense lesion material properties to be assigned before FEA to their respective lesions. A future goal is to include correlation between DBT and ABUS sets in the axilla region of the breast.

When translating this technique to patients, we intend to use more external markers placed at better breast locations and have the patient wear a special camisole for the ABUS acquisitions. Currently, this study shows satisfactory results with the use of just three external markers for both phantoms. The use of more external markers will allow for full coverage of the breast including the axillary region and we can better determine what number of markers and their locations render the best results. As shown for the results of Phantom B, it would be better to directly determine which marker locations are best for all patient data using the same number of markers at the same locations and then using the algorithm to determine the minimum needed for a desired registration result.

We expect that with the use of a breast ultrasound camisole, breast movement between various scans can be restricted. This restriction should reduce the effect of varying the compression between ABUS views. The use of the camisole can also address an additional potential problem with the external markers becoming attached to the ultrasound mesh paddle between multiple ABUS compressions. This camisole would need to be made of sheer material similar to the Invenia mesh membrane that does not affect the ultrasound depth of penetration and also does not interfere with coupling to the ultrasound transducer. Ultrasound penetration and coupling of the Invenia mesh material is well understood and in some cases the use of this material improves coupling as it holds ultrasound gel in place. The SonoCiné AWBUS system (SonoCiné, Inc., Reno, NV) ${ }^{48}$ is a commercially available system that uses an ultrasound camisole that fits similar to a sports bra to reduce breast movement during scanning. By restricting breast motion, a breast camisole should allow for improvement in lesion registration for the deformable mapping algorithm.

Specifically, even if some markers become detached there will still be a sufficient number remaining that can be used for registration. This study uses a tight $15 \mathrm{~mm}$ bound when determining a match between corresponding lesions since the phantoms have a uniform background. When translated to real breasts, this bound will likely increase due to breast heterogeneity and breast structures (i.e., Cooper's ligaments) that will not be modeled in the FE process. In addition, we expect to segment glandular tissue from the background adipose tissue in the breast of the ABUS ${ }^{41}$ and DBT images. Several studies, have found successful results in determining breast density from DBT breast images. ${ }^{42-44}$ Although, the poor spatial resolution of DBT in the $\mathrm{z}$ (depth) direction reduces the accuracy of the dense tissue segmentation in that direction, the overall coarse volumetric segmentation should still be of value in providing information about the spatial distribution of the glandular and adipose tissues within the breast for the deformable mapping. This should assist with the lesion correlation when the method is translated to patient images.

The process that took the most time in this study was the manual segmentation. In order for this technique to be effective in a clinical platform semi-automated and automated segmentation techniques will be needed and explored. Once all images are segmented the automated deformable mapping algorithm takes about $40 \mathrm{~min}$ to run on a Windows 7 Intel ${ }^{\circledR}$ Core $^{\mathrm{TM}} \mathrm{i} 7 \mathrm{CPU}$ with a speed of $2500 \mathrm{MHz}$ and 4 GB RAM. The runtime could be improved greatly with a computer with more memory and a capable GPU.

\section{CONCLUSIONS}

This work demonstrates the potential use of deformable mapping techniques to relate lesions between DBT and ABUS breast images. The utilization of external fiducial markers has been shown to improve the accuracy of this approach. The resulting one-to-one correlation between lesions in DBT and ABUS could help improve radiologists' characterization of breast lesions, which can reduce patient callbacks, negative biopsies and false negative biopsies. 
Future work will expand this platform to include an IRB approved study for patient volunteers, and an expansion of the deformable mapping technique for use in relating lesions in other breast modalities such as MRI, dedicated breast CT and transmission US.

\section{ACKNOWLEDGMENTS}

This work was supported in-part by a research grant (15PAF04328) from GE Global Research. Crystal A. Green is supported by The Science, Mathematics and Research for Transformation (SMART) Scholarship for Service Program (HQ0034-16-C-0008). The authors would like to thank Ted Lynch, Ph.D, of CIRS, Inc. for his assistance in phantom development and characterization. The authors would like to thank Darius Fadanelli, Eamon Whalen, and John P. Kytasty of Altair Engineering Inc. for their assistance in FE modeling and processing.

\section{CONFLICTS OF INTEREST}

M. Goodsitt is a co-investigator on a grant funded by GE Healthcare. M. Goodsitt and P. Carson are collaborators on research with GE Global Research, Niskayuna, NY. C. Davis is an employee of General Electric Corporation and holds several U.S. patents on medical imaging.

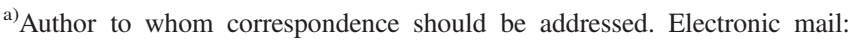
canngree@umich.edu.
}

\section{REFERENCES}

1. Jackson V. The role of US in breast imaging. Radiology. 1990;177:305311.

2. Taylor KJW, Merritt C, Piccoli C, et al. Breast examination to characterize breast masses. Ultrasound Med Biol. 2002;28:19-26.

3. Ohuchi N, Suzuki A, Sobue T, et al. Sensitivity and specificity of mammography and adjunctive ultrasonography to screen for breast cancer in the Japan Strategic Anti-cancer Randomized Trial (J-START): a randomised controlled trial. Lancet. 2016;387:341-348.

4. Wilczek B, Wilczek HE, Rasouliyan L, Leifland K. Adding 3D automated breast ultrasound to mammography screening in women with heterogeneously and extremely dense breasts: report from a hospitalbased, high-volume, single-center breast cancer screening program. Eur J Radiol. 2016;85:1554-1563.

5. Giger ML, Inciardi MF, Edwards A, et al. Automated breast ultrasound in breast cancer screening of women with dense breasts: reader study of mammography-negative and mammography-positive cancers. Am J Roentgenol. 2016;206:1341-1350.

6. Brem RF, Tabár L, Duffy SW, et al. Assessing improvement in detection of breast cancer with three-dimensional automated breast us in women with dense breast tissue: the somoinsight study. Radiology. 2015;274: 663-673.

7. Hakim CM, Chough DM, Ganott MA, et al. Digital breast tomosynthesis in the diagnostic environment: a subjective side-by-side review. Am J Roentgenol. 2010;195:W172-W176.

8. Kim SA, Chang JM, Cho N, Yi A, Moon WK. Characterization of breast lesions: comparison of digital breast tomosynthesis and ultrasonography. Korean J Radiol. 2015;6:229-238.

9. Conway WF, Hayes CW, Brewer WH. Occult breast masses: use of a mammographic localizing grid for US evaluation. Radiology. 1991;181:143-146.
10. Sinha SP, Goodsitt MM, Roubidoux MA, et al. Automated ultrasound scanning on a dual-modality breast imaging system: coverage and motion issues and solutions. J Ultrasound Med. 2007;26:645-655.

11. Sinha SP, Roubidoux MA, Helvie MA, et al. Multi-modality 3d breast imaging with X-ray tomosynthesis and automated ultrasound. In: Proceedings of the 29th Annual International Conference of the IEEE EMBS; 2007:1335-1338.

12. Goodsitt MM, Chan HP, Hadjiiski L, LeCarpentier GL, Carson PL. Automated registration of volumes of interest for a combined X-ray tomosynthesis and ultrasound breast imaging system. Lect Notes Comput Sci (including Subser Lect Notes Artif Intell Lect Notes Bioinformatics); 2008, vol. 5116 LNCS:463-468.

13. Kapur A, Carson PL, Eberhard J, et al. Combination of digital mammography with semi-automated 3D breast ultrasound. Technol Cancer Res Treat. 2004;3:325-334.

14. Padilla F, Roubidoux MA, Paramagul C, et al. Breast mass characterization using 3-dimensional automated ultrasound as an adjunct to digital breast. Am Inst Ultrasound Med. 2013;32:93-104.

15. Lecarpentier GL, Goodsitt MM, Verweij S, Li J, Padilla F, Carson PL. Acoustic performance of mesh compression paddles for a multimodality breast imaging system. Ultrasound Med Biol. 2014;40: 1503-1511.

16. Li J, Goodsitt MM, Padilla F, et al. Effect of a gel retainment dam on automated ultrasound coverage in a dual-modality breast imaging ultrasound system. J Ultrasound Med. 2010;29:1075-1081.

17. Vaughan CL, Douglas TS, Said-Hartley Q, et al. Testing a dual-modality system that combines full-field digital mammography and automated breast ultrasound. Clin Imaging. 2016;40:498-505.

18. Schulz-Wendtland R, Jud SM, Fasching PA, et al. A standard mammography unit - standard 3D ultrasound probe fusion prototype: first results. Geburtshilfe Frauenheilkd. 2017;77:679-685.

19. Larson ED, Lee W-M, Roubidoux MA, et al. Automated breast ultrasound: dual-sided compared with single-sided imaging. Ultrasound Med Biol. 2016;42:2072-2082.

20. Samani A, Bishop J, Yaffe MJ, Plewes DB. Biomechanical 3-D finite element modeling of the human breast using MRI data. IEEE Trans Med Imaging. 2001;20:271-279.

21. Han L, Hipwell JH, Tanner C, et al. Development of patient-specific biomechanical models. Phys Med Biol. 2012;57:455-472.

22. Tanner C, Guarino S, Hall-craggs MA, Hawkes DJ. Large breast compressions: observations and evaluation of simulations. Med Phys. 2011;38:682-690.

23. Mertzanidou T, Hipwell J, Johnsen S, et al. MRI to X-ray mammography intensity-based registration with simultaneous optimisation of pose and biomechanical transformation parameters. Med Image Anal. 2014;18:674-683.

24. Lee AWC, Rajagopal V, Babarenda Gamage TP, Doyle AJ, Nielsen PMF, Nash MP. Breast lesion co-localisation between X-ray and MR images using finite element modelling. Med Image Anal. 2013;17:1256-1264.

25. Solves-Llorens JA, Rupérez MJ, Monserrat C. A complete software application for automatic registration of x-ray mammography and magnetic resonance images. Med Phys. 2014;41:081903.

26. Hopp T, Dietzel M, Baltzer PA, et al. Automatic multimodal 2D/3D breast image registration using biomechanical FEM models and intensity-based optimization. Med Image Anal. 2013;17:209-218.

27. Hipwell JH, Vavourakis V, Han L, Mertzanidou T, Eiben B, Hawkes DJ. A review of biomechanically informed breast image registration. Phys Med Biol. 2016;61:R1-R31.

28. Georgii J, Zöhrer F, Hahn HK. Model-based position correlation between breast images. In: Proceedings Volume 8670, Medical Imaging 2013: Computer-Aided Diagnosis. Vol 8670; 2013:1-7.

29. Ruiter NV, Stotzka R, Müller T, Gemmeke H, Reichenbach JR, Kaiser WA. Model-based registration of X-ray mammograms and MR images of the female breast. IEEE Trans Nucl Sci. 2006;53:204-211.

30. Telegrafo M, Rella L, Stabile Ianora AA, Angelelli G, Moschetta M. Supine breast US: how to correlate breast lesions from prone MRI. $\mathrm{Br} J$ Radiol. 2016;89:20150497.

31. Hopp T, Duric N, Ruiter NV. Computerized medical imaging and graphics image fusion of ultrasound computer tomography volumes with $\mathrm{X}$ ray mammograms using a biomechanical model based 2D/3D registration. Comput Med Imaging Graph. 2015;40:170-181. 
32. Narayanasamy G, LeCarpentier GL, Roubidoux M, Fowlkes JB, Schott AF, Carson PL. Spatial registration of temporally separated whole breast 3D ultrasound images. Med Phys. 2009;36:4288.

33. Krol A, Unlu MZ, Baum KG, et al. MRI/PET nonrigid breast-image registration using skin fiducial markers. Phys Med. 2006;21:39-43.

34. Unlu MZ, Krol A, Magri A, et al. Computerized method for nonrigid MR-to-PET breast-image registration. Comput Biol Med. 2010;40:37-53.

35. Kucukkaya F, Aribal E, Tureli D, Altas H, Kaya H. Use of a volume navigation technique for combining real-time ultrasound and contrastenhanced MRI: accuracy and feasibility of a novel technique for locating breast lesions fikret kucukkaya1. Am J Roentgenol. 2016;206: 217-225.

36. Hungr N, Long JA, Beix V, Troccaz J. A realistic deformable prostate phantom for multimodal imaging and needle-insertion procedures. Med Phys. 2012;39:2031-2041.

37. Negron LA, Viola F, Black EP, Toth CA, Walker WF. Development and characterization of a radiation force imaging. IEEE Trans Ultrason Ferroelectr Freq Control. 2002;49:1543-1551.

38. Howard S, Yuen J, Wegner P, Zanelli CI. Characterization and FEA simulation for a HIFU phantom material. IEEE Symp Ultrason 2003. 2003;2(c):1270-1273.

39. Adam Cunha J, Hsu IC, Pouliot J, et al. Toward adaptive stereotactic robotic brachytherapy for prostate cancer: demonstration of an adaptive workflow incorporating inverse planning and an MR stealth robot. Minim Invasive Ther Allied Technol. 2010;19:189-202.

40. Cloutier G, Soulez G, Qanadli SD, et al. A multimodality vascular imaging phantom with fiducial markers visible in DSA, CTA, MRA, and ultrasound. Med Phys. 2004;31:1424-1433.

41. Mundinger A. 3D supine automated ultrasound (SAUS, ABUS, ABVS) for supplemental screening women with dense breasts. J Breast Heal. 2016;12:52-55.

42. Stavros AT, Rapp CL, Parker SH. Breast Ultrasound. Philadelphia, PA: Lippincott Williams \& Wilkins; 2004.
43. Fedorov A, Beichel R, Kalpathy-cramer J, et al. 3D Slicer as an image computing platform for the quantitative imaging network. Magn Reson Imaging. 2012;30:1323-1341.

44. Dice LR. Measures of the amount of ecologic association between species. Ecology. 1945;26:297-302.

45. Brock KK, Sharpe MB, Dawson LA, Kim SM, Jaffray DA. Accuracy of finite element model-based multi-organ deformable image registration. Med Phys. 2005;32:1647-1659.

46. Boverman G, Davis CEL, Geimer SD, Meaney PM. Image Registration for Microwave Tomography of the Breast Using Priors from Non-Simultaneous Previous Magnetic Resonance Images. In: 2017 First IEEE MTT-S International Microwave Bio Conference (IMBIOC). Vol 7249. IEEE; 2017:1-4.

47. Gu P, Lee W-M, Roubidoux MA, Yuan J, Wang X, Carson PL. Automated 3D ultrasound image segmentation to aid breast cancer image interpretation. Ultrasonics. 2016;65:51-58.

48. Kelly KM, Richwald GA. Automated whole-breast ultrasound: advancing the performance of breast cancer screening. Semin Ultrasound CT MRI. 2011;32:273-280.

\section{SUPPORTING INFORMATION}

Additional supporting information may be found online in the Supporting Information section at the end of the article.

Data S1. Pseudo-code for lesion correlation used within deformable mapping algorithm.

Data S2. Load-strain curve comparing elastic properties of Phantom A and Phantom B.

Data S3. Periphery uncertainty from skin segmentation for a slice in ABUS for (a) Phantom A (b) Phantom B. 